\title{
The Efimov Effect. Discrete Spectrum Asymptotics
}

\author{
A.V. Sobolev \\ Université Paris-Nord, Département de Mathématiques et Informatique, 93430 Villetaneuse, \\ France and St-Petersburg Branch of the Steklov Institute (SPOMI), St-Petersburg, Russia, \\ 191011. Current address: Department of Mathematics, University of Toronto, Toronto, Canada \\ M5S 1A1
}

Received June 19, 1992; in revised form November 11, 1992

\begin{abstract}
We study a three-particle Schrödinger operator $H$ for which none of the two-particle subsystems has negative bound states and at least two of them have zero energy resonances. We prove that under this condition the number $N(z)$ of bound states of $H$ below $z<0$ has the asymptotics $N(z) \sim \mathfrak{U}_{0}|\log | z||$ as $z \rightarrow-0$, where the coefficient $\mathfrak{U}_{0}$ depends only on the ratio of masses of the particles.
\end{abstract}

\section{Introduction}

We are going to discuss the following remarkable phenomenon of the spectral theory of the three-body Schrödinger operators, known as the Efimov effect. Let $h_{\alpha}$, $\alpha=1,2,3$, be Hamiltonians describing two-particle subsystems of a three-particle system with the internal short-range potentials $v_{\alpha}(x), x \in \mathbb{R}^{3}$. Suppose that none of $h_{\alpha}$ has negative eigenvalues and at least two of the hamiltonians $h_{\alpha}$ have zero energy resonances. Then the three-particle operator $H$ will have infinitely many negative eigenvalues accumulating at zero. Below we denote by $N(z), z<0$, the number of eigenvalues of $H$ lying on the left from the point $z$. For the first time the Efimov effect has been discussed in [4]. An independent proof on a physical level of rigor has been also given in [2]. The first rigorous proof has been presented in paper [12]. An alternative approach for spherically symmetric potentials $v_{\alpha}$ has been put forward in [10]. The growth of $N(z)$ as $z \rightarrow-0$ has been studied in paper [1] for the symmetric case. Namely, the authors of [1] have found the exponential asymptotics of eigenvalues corresponding to spherically symmetric bound states. This result is consistent with the lower bound

$$
\lim _{z \rightarrow-0} \inf |\log | z||^{-1} N(z)>0
$$

established in [11] without any symmetry assumptions.

The aim of the present paper is to study the asymptotics of $N(z)$ as $z \rightarrow-0$. We do not assume that the pair potentials $v_{\alpha}$ are symmetric but suppose that $v_{\alpha} \leqq 0$. 
Our main result is the asymptotics (see Theorem 3.1)

$$
\lim _{z \rightarrow-0} \inf |\log | z||^{-1} N(z)=\mathfrak{U}_{0},
$$

where the coefficient $\mathfrak{U}_{0}$ does not depend on the pair potentials $v_{\alpha}$ and is a positive function of the ratios $m_{1} / m_{2}, m_{2} / m_{3}$ of the masses of three particles under consideration. The explicit formula for $\mathfrak{U}_{0}$ is given in Sect. 3. Note that (1.2) confirms the sharpness of the bound (1.1).

Let us give a sketch of the main ideas of the proof of (1.2). As in [12], we reduce the problem to the study of the compact selfadjoint operator $\mathbf{A}(z)$ acting in $L_{2}^{(3)}(\mathfrak{N})$ $(\mathfrak{N}$ is the configuration space of the three-particle system) which is a symmetrized analog of the operator entering the Faddeev equations (see [6] and also [7]). We rely upon the relation (see Theorem 4.1)

$$
N(z, H)=n(1, \mathbf{A}(z)),
$$

where $n(\mu, B)$ denotes the number of eigenvalues of the compact selfadjoint operator $B$ lying on the right from the point $\mu>0$. To study the behaviour of $\mathbf{A}(z)$ for small $z$ we establish the asymptotics of the resolvent $r_{\alpha}(z)$ of the two-particle operator $h_{\alpha}$ as $z \rightarrow-0$ under the condition that $h_{\alpha}$ has the zero energy resonance (see Lemma 2.2). To that end we use a simplified version of the technique developed in [9]. Lemma 2.2 enables us to single out the leading term of the operator $\mathbf{A}(z)$ as $z \rightarrow-0$. It proves to be unitarily equivalent to a compact integral operator $\mathbf{S}_{R}$, $R=1 / 2|\log | z||$, with a Toeplitz type kernel, acting in $L_{2}\left((0, R), L_{2}^{(3)}\left(\mathbb{S}^{2}\right)\right)$. This allows to find the asymptotics of $n\left(1, \mathbf{S}_{R}\right)$ as $R \rightarrow \infty$ by employing a standard argument known as the calculation of the canonical distribution of a Toeplitz operator (see [8]). As the result we get (see Theorem 4.5):

$$
\lim _{R \rightarrow \infty}(2 R)^{-1} n\left(1, \mathbf{S}_{R}\right)=\mathfrak{U}_{0},
$$

which in combination with (1.3) yields (1.2). The plan of the paper is as follows. In Sect. 2 we obtain the asymptotics as $z \rightarrow-0$ of the two-particle resolvent assuming the presence of the zero energy resonance. The precise formulation of the main result (Theorem 3.1) and its discussion are given in Sect. 3. In Sect. 4 we establish the relation (1.3), study the operator $\mathbf{A}(z)$ as $z \rightarrow-0$ and prove Theorem 3.1. Some technical material is collected in Appendix.

Throughout the paper we adopt the following conventions. We say that an operator valued function is continuous if it is continuous in the norm sense. The scalar products in $L_{2}(\cdot)$ and $\mathbb{R}^{3}$ are denoted by $(\cdot, \cdot)$ and $\langle\cdot, \cdot\rangle$ respectively. The integrals with no indication of the limits imply the integration over the whole space. By $C$ and $c$ we denote various positive constants whose exact values are of no importance.

\section{Two-Particle Schrödinger Operator}

In this section we study a two-particle system. Let $h_{0}=-(2 m)^{-1} \Delta, h=h_{0}+v$ in $\mathfrak{H}=L_{2}\left(\mathbb{R}^{3}\right)$. Here $m>0$ is the reduced mass of the system, $v$ is a real-valued potential, satisfying the condition

$$
|v(x)| \leqq C(1+|x|)^{-b}, \quad b>3 .
$$


By $r_{0}(z), r(z)$ we denote the resolvents of $h_{0}$ and $h$ respectively. Note the identity

$$
r=r_{0}-r_{0} v r=r_{0}-r v r_{0} \text {. }
$$

Denote $v^{\frac{1}{2}}=v|v|^{-\frac{1}{2}}$

$$
w(z)=I-|v|^{\frac{1}{2}} r(z) v^{\frac{1}{2}}
$$

Then (2.2) immediately yields

$$
w(z)=\left(I+|v|^{\frac{1}{2}} r_{0}(z) v^{\frac{1}{2}}\right)^{-1} .
$$

Recall the explicit expressions for the kernel of $r_{0}(z)$ :

$$
r_{0}\left(x, x^{\prime} ; z\right)=\frac{m}{2 \pi} \frac{e^{i \sqrt{2 m z}\left|x-x^{\prime}\right|}}{\left|x-x^{\prime}\right|}, \quad \operatorname{Im} \sqrt{z} \geqq 0 .
$$

In what follows we need an asymptotic resolution of the operator $|v|^{\frac{1}{2}} r_{0}(z) v^{\frac{1}{2}}$, $z=-k^{2}, k>0$, near $k=0$. Denote by $G_{0}, G_{1}$ the operators with the kernels

$$
\begin{aligned}
G_{0}\left(x, x^{\prime}\right) & =\frac{m}{2 \pi} \frac{|v|^{\frac{1}{2}}(x) v^{\frac{1}{2}}\left(x^{\prime}\right)}{\left|x-x^{\prime}\right|}, \\
G_{1}\left(x, x^{\prime}\right) & =\frac{m^{\frac{3}{2}}}{2^{\frac{1}{2}} \pi}|v|^{\frac{1}{2}}(x) v^{\frac{1}{2}}\left(x^{\prime}\right) .
\end{aligned}
$$

We have the following

Lemma 2.1. Let $v$ satisfy (2.1). Then for any positive $\delta<\min \{1,(b-3) / 2\}$ the relation holds

$$
|v|^{\frac{1}{2}} r_{0}\left(-k^{2}\right) v^{\frac{1}{2}}=G_{0}-k G_{1}+k^{1+\delta} G_{2}^{(\delta)}(k),
$$

where $G_{2}^{(\delta)}(k)$ is continuous in $k \geqq 0$.

Proof. Set $f(t ; s):=(4 \pi t)^{-1}\left(e^{-s t}-1+s t\right), t>0$, and introduce the operator $G_{2}(k)$ with the kernel

$$
2 m|v|^{\frac{1}{2}}(x) f\left(x-x^{\prime} ; k \sqrt{2 m}\right) v^{\frac{1}{2}}\left(x^{\prime}\right) .
$$

In view of (2.5) we have formally

$$
|v|^{\frac{1}{2}} r_{0}\left(-k^{2}\right) v^{\frac{1}{2}}=G_{0}-k G_{1}+G_{2}(k) .
$$

Since $f(t ; s) \leqq C_{\delta} s^{1+\delta} t^{\delta}$ for any $\delta \in(0,1]$, the kernel of $G_{2}(k)$ does not exceed

$$
C_{\delta} k^{1+\delta}|v|^{\frac{1}{2}}(x)|v|^{\frac{1}{2}}\left(x^{\prime}\right)\left(|x|^{\delta}+\left|x^{\prime}\right|^{\delta}\right) .
$$

Choosing $\delta<(b-3) / 2$, we see that $G_{2}(k)$ is Hilbert-Schmidt and the operator $G_{2}^{(\delta)}(k):=k^{-1-\delta} G_{2}(k)$ is continuous in $k \geqq 0$.

From now on we suppose that $v \leqq 0$, so that $v^{\frac{1}{2}}=-|v|^{\frac{1}{2}}$. All our arguments in this section go through for arbitrary $v$ as well but in the next sections we look at nonpositive potentials only.

Now we are going to describe the behaviour of the operator $w\left(-k^{2}\right)$ as $k \rightarrow 0$. Note that under the condition (2.1) the operator $h$ has finite discrete spectrum, so that the resolvent $r\left(-k^{2}\right)$ and, consequently, $w\left(-k^{2}\right)$, is well defined for small $k$. We deal with one of the two following situations. 
(1) The point -1 is a simple eigenvalue of the operator $G_{0}$ and the corresponding eigenfunction $\varphi$ satisfies the condition $\int|v|^{\frac{1}{2}}(x) \varphi(x) d x \neq 0$. To be definite we normalize $\varphi$ so that

$$
\left(\varphi,|v|^{\frac{1}{2}}\right)=2^{\frac{1}{4}} \pi^{\frac{1}{2}} m^{-\frac{3}{4}} .
$$

In this situation we say that zero is a resonance of $h$ or $h$ has a zero energy resonance. One can prove (see [9]) that the function

$$
u(x)=\int \frac{|v|^{\frac{1}{2}}\left(x^{\prime}\right)}{\left|x-x^{\prime}\right|} \varphi\left(x^{\prime}\right) d x^{\prime}
$$

is a unique solution (up to a factor) of the Schrödinger equation $h u+v u=0$ in $L^{2}$-space with the weight $\left(1+x^{2}\right)^{-s / 2}, \forall s>1 / 2$, and $u \notin \mathfrak{H}$.

Note an important property of $\varphi$. Set

$$
\psi(k)=(2 \pi)^{-\frac{3}{2}} \int e^{-i k x}|v(x)|^{\frac{1}{2}} \varphi(x) d x .
$$

This function obeys the estimate

$$
|\psi(k)-\psi(0)| \leqq C_{\delta}|k|^{\delta}, \quad 0<\delta<\frac{b-3}{2} .
$$

Indeed, since $\left|e^{-i k x}-1\right| \leqq|k|^{\delta}|x|^{\delta}$, we have

$$
|\psi(k)-\psi(0)| \leqq|k|^{\delta}\left(\int|x|^{2 \delta}|v(x)| d x\right)^{\frac{1}{2}}\left(\int|\varphi(x)|^{2} d x\right)^{\frac{1}{2}} .
$$

The r.h.s. is finite for $\delta<(b-3) / 2$.

(2) The point -1 is not the eigenvalue of the operator $G_{0}$. Then one can prove that $\lambda=0$ is neither resonance nor eigenvalue of $h$. So it is natural to say that zero is a regular point of $h$.

We do not discuss here the other possible cases: zero is the eigenvalue of $h$ or the eigenvalue and the resonance at the same time.

Lemma 2.2. Let $v$ obeys (2.1) and $k>0$ be small enough, so the operator $w\left(-k^{2}\right)$ is defined.

(1) If zero is a regular point of $h$ then $w\left(-k^{2}\right)$ is continuous in $k \geqq 0$.

(2) If zero is a resonance of $h$ then for any positive $\delta<1 / 2 \min \{1, b-3\}$ the representation

$$
w\left(-k^{2}\right)=\frac{(\cdot, \varphi) \varphi}{k}+k^{-1+\delta} w^{(\delta)}(k)
$$

is valid, where the operator $w^{(\delta)}(k)$ is continuous in $k \geqq 0$. If, in addition, $h \geqq 0$ then $w\left(-k^{2}\right) \geqq 0$ and

$$
\left(w\left(-k^{2}\right)\right)^{\frac{1}{2}}=\frac{(\cdot, \varphi) \varphi}{a k^{\frac{1}{2}}}+k^{-\frac{1-\delta}{2}} \tilde{w}^{(\delta)}(k), \quad a=\|\varphi\|,
$$

where the operator $\tilde{w}^{(\delta)}(k)$ is continuous in $k \geqq 0$.

Proof. (1) Let zero be a regular point. Then according to (2.4) and (2.7),

$$
w\left(-k^{2}\right)=\left(I+G_{0}+o(1)\right)^{-1}=\left(I+G_{0}\right)^{-1}+o(1), \quad k \rightarrow 0,
$$

which gives the desired result. 
(2) Let zero be a resonance. Denote by $P_{0}$ the one-dimensional projector onto the subspace associated with $\varphi$ and by $P_{1}$ the projector onto its orthogonal complement, so that $P_{0} \oplus P_{1}=I$. Let us write the operator $A=A(k)=$ $I-|v|^{\frac{1}{2}} r_{0}\left(-k^{2}\right)|v|^{\frac{1}{2}}$ in the matrix form:

$$
A=\left(\begin{array}{ll}
A_{00} & A_{01} \\
A_{10} & A_{11}
\end{array}\right),
$$

where $A_{j k}=P_{j} A P_{k}: P_{k} \mathfrak{H} \rightarrow P_{j} \mathfrak{H}, k, j=0,1$. It is more convenient instead of $A$ to consider the operator

$$
B=P A P, \quad P:=\left(\begin{array}{cc}
k^{-\frac{1}{2}} P_{0} & 0 \\
0 & P_{1}
\end{array}\right),
$$

By (2.7) its entries are

$$
\begin{aligned}
& B_{00}=-P_{0} G_{1} P_{0}+k^{\delta} P_{0} G_{2}^{(\delta)}(k) P_{0}, \\
& B_{01}=-k^{\frac{1}{2}} P_{0}\left[G_{1}-k^{\delta} G_{2}^{(\delta)}(k)\right] P_{1}, \\
& B_{10}=B_{01}^{*} \\
& B_{11}=P_{1}\left(I+G_{0}\right) P_{1}-k P_{1}\left[G_{1}-k^{\delta} G_{2}^{(\delta)}(k)\right] P_{1}
\end{aligned}
$$

with $\delta<\min \{1,(b-3) / 2\}$. Therefore $B=B^{(0)}+K$, where

$$
B^{(0)}=\left(\begin{array}{cc}
-P_{0} G_{1} P_{0} & 0 \\
0 & P_{1}\left(I+G_{0}\right) P_{1}
\end{array}\right)
$$

and $K=O\left(k^{\gamma}\right), \quad \gamma=\min \{1 / 2, \delta\}$. By the definition of $P_{1}$ the operator $F=\left(P_{1}\left(I+G_{0}\right) P_{1}\right)^{-1}$ exists in $P_{1} \mathfrak{H}$. Furthermore, taking into account the equalities (2.8) and $P_{0}=a^{-2}(\cdot, \varphi) \varphi$, one can obtain from (2.6) that

$$
-P_{0} G_{1} P_{0}=P_{0} \frac{m^{\frac{3}{2}}}{2^{\frac{1}{2}} \pi}\left(\varphi,|v|^{\frac{1}{2}}\right)^{2} a^{-2}=a^{-2} P_{0}
$$

Thus $\left(-P_{0} G_{1} P_{0}\right)^{-1}=a^{2} P_{0}=(\cdot, \varphi) \varphi$. Now since $B=\left(I+K\left(B^{(0)}\right)^{-1}\right) B^{(0)}$ and $K=O\left(k^{\gamma}\right)$ as $k \rightarrow 0$, we have

$$
B^{-1}=\left(B^{(0)}\right)^{-1}+O\left(k^{\gamma}\right)=\left(\begin{array}{cc}
(\cdot, \varphi) \varphi & 0 \\
0 & F
\end{array}\right)+O\left(k^{\gamma}\right) .
$$

Taking into account that $w\left(-k^{2}\right)=(A(k))^{-1}=P B^{-1} P$, we complete the proof of (2.11).

Let us prove (2.12). Since $r\left(-k^{2}\right) \geqq 0$ for $h \geqq 0$ we have $w\left(-k^{2}\right) \geqq I \geqq 0$. Further, note that

$$
\left(\frac{(\cdot, \varphi) \varphi}{k}\right)^{\frac{1}{2}}=\frac{(\cdot, \varphi) \varphi}{a k^{\frac{1}{2}}}
$$

and recall the well known inequality for arbitrary positive operators $A, B$ (see [3]):

$$
\left\|B^{\frac{1}{2}}-A^{\frac{1}{2}}\right\| \leqq\|B-A\|^{\frac{1}{2}} .
$$


In combination with (2.11) it yields

$$
\left\|\left(w\left(-k^{2}\right)\right)^{\frac{1}{2}}-\frac{(\cdot, \varphi) \varphi}{a k^{\frac{1}{2}}}\right\| \leqq C k^{-\frac{1-\delta}{2}} .
$$

This gives (2.12).

We point out that a decomposition of the type (2.11) was proven for the first time in [13] (see also [12]). The idea of our proof of Lemma 2.2 is borrowed from the more recent paper [9], where an asymptotic expansion similar to (2.11) was obtained (see Lemma 4.3 in [9]). However the formula from [9] provides the asymptotic expansion with further terms up to the order $o(k)$ which calls for the condition $b>5$ in (2.1). It is sufficient for us to have much more rough result (2.11) which is valid for $b>3$.

In Sect. 4 it will be convenient to write down the operator $\left(w\left(-k^{2}\right)\right)^{1 / 2}$ in the form (2.12) not only for small $k$ but for all $k \geqq 0$. Namely, let $\zeta \in C^{\infty}\left(\mathbb{R}_{+}\right)$be a function such that $\zeta(t)>0$ for all $t>0, \zeta(t)=t, t \leqq 1$ and $\zeta(t)=1, t \geqq 2$. Then for all $k \geqq 0$ we have

$$
\left(w\left(-k^{2}\right)\right)^{\frac{1}{2}}=\frac{(\cdot, \varphi) \varphi}{a k^{\frac{1}{2}}}+(\zeta(k))^{-\frac{1-\delta}{2}} \tilde{w}^{(\delta)}(k), \quad a=\|\varphi\|,
$$

where the operator $\tilde{w}^{(\delta)}(k)$ is uniformly bounded and continuous in $k \geqq 0$.

\section{The Main Result and its Discussion}

1. We consider a system of three particles with the masses $m_{1}, m_{2}, m_{3}$, one of them may be infinite. We always work in the system with the removed center of mass motion, so the configuration space is a six-dimensional subspace $\mathfrak{N}$ of $\mathbb{R}^{9}$. In contrast to the two-particle case we use as a rule the momentum representation, that is we use one of the three pairs of coordinates $\left(k_{\alpha}, p_{\alpha}\right)$ conjugate to the conventional Jacoby coordinates $\left(x_{\alpha}, y_{\alpha}\right)$. The subscript $\alpha$ is equal either to 1 or 2 or 3. Sometimes instead of $\left(k_{\alpha}, p_{\alpha}\right)$ we use one of the pairs $\left(p_{\alpha}, p_{\beta}\right)$. Various coordinates in $\mathfrak{N}$ are related as follows:

$$
p_{1}+p_{2}+p_{3}=0, \quad \pm k_{\alpha}=m_{\beta}\left(m_{\beta}+m_{\gamma}\right)^{-1} p_{\alpha}+p_{\beta} .
$$

Here and below we always assume that $\alpha \neq \beta, \beta \neq \gamma, \alpha \neq \gamma$. The sign " + " (or "- ") corresponds to the case $\beta<\alpha$ (or $\alpha<\beta$ ). For brevity we often use the notation $k_{\alpha}=d_{\alpha \beta} p_{\alpha}+e_{\alpha \beta} p_{\beta}$, where the coefficients $d_{\alpha \beta}$ and $e_{\alpha \beta}$ can be expressed explicitly via $m_{\alpha}, m_{\beta}, m_{\gamma}$ by means of (3.1). In certain cases it is convenient to use the "mixed" coordinates $\left(x_{\alpha}, p_{\alpha}\right)$. The transition to $\left(k_{\alpha}, p_{\alpha}\right)$ is performed by the "partial" Fourier transform:

$$
\left(\Phi_{\alpha} f\right)\left(k_{\alpha}, p_{\alpha}\right)=(2 \pi)^{-\frac{3}{2}} \int e^{-i k_{\alpha} x_{\alpha}} f\left(x_{\alpha}, p_{\alpha}\right) d x_{\alpha} .
$$

The three-particle Schrödinger operator has the form

$$
H=H_{0}+\sum_{\alpha} V_{\alpha}
$$


where

$$
H_{0} f\left(k_{\alpha}, p_{\alpha}\right)=H^{0}\left(k_{\alpha}, p_{\alpha}\right) f\left(k_{\alpha}, p_{\alpha}\right), \quad H^{0}(k, p)=\frac{k^{2}}{2 l_{\alpha}}+\frac{p^{2}}{2 n_{\alpha}} .
$$

Here $l_{\alpha}, n_{\alpha}$ are the reduced masses:

$$
l_{\alpha}=m_{\beta} m_{\gamma}\left(m_{\beta}+m_{\gamma}\right)^{-1}, \quad n_{\alpha}=m_{\alpha}\left(m_{\beta}+m_{\gamma}\right) M^{-1}, \quad M=m_{1}+m_{2}+m_{3} .
$$

The interactions $V_{\alpha}$ are given by the operators:

$$
V_{\alpha}=\Phi_{\alpha} v_{\alpha} \Phi_{\alpha}^{*}
$$

where $v_{\alpha}, \alpha=1,2,3$, are multiplications by bounded real valued functions $v_{\alpha}\left(x_{\alpha}\right)$ (pair potentials in the spatial representation). For example, $v_{3}$ describes the interaction of the first and second particles. We suppose that the functions $v_{\alpha}$ satisfy (2.1). Note that the function $H^{0}\left(k_{\alpha}, p_{\alpha}\right)$ in fact does not depend on the particular choice of $\alpha$. We denote by $H_{\alpha \beta}^{0}$ the function $H^{0}$ expressed in terms of $p_{\alpha}, p_{\beta}$, i.e. $H_{\alpha \beta}^{0}(p, q)=H^{0}\left(d_{\alpha \beta} p+e_{\alpha \beta} q, q\right)$. By means of (3.1) one can easily prove that

$$
H_{\alpha \beta}^{0}(p, q)=\frac{p^{2}}{2 l_{\beta}}+\frac{\langle p, q\rangle}{m_{\gamma}}+\frac{q^{2}}{2 l_{\alpha}} .
$$

By virtue of (3.3) it follows from here that

$$
H_{\alpha \beta}^{0}(p, q) \geqq \frac{p^{2}}{2 m_{\alpha}}+\frac{q^{2}}{2 m_{\beta}} .
$$

Two-particle subsystems are described by Hamiltonians $h_{\alpha}=-\left(2 l_{\alpha}\right)^{-1} \Delta_{x_{\alpha}}$ $+v_{\alpha}$ in $L_{2}\left(\mathbb{R}^{3}\right)$. For the other two-particle objects we use the notations introduced in Sect. 2 but endue them with the subscript $\alpha$. For example, $w_{\alpha}$ means the operator (2.3) for the two-particle subsystem $\alpha$. If the subsystem has a zero energy resonance we normalize the corresponding function $\varphi_{\alpha}$ in agreement with (2.8):

$$
(2 \pi)^{\frac{3}{2}} \psi_{\alpha}(0)=\left(\varphi_{\alpha},\left|v_{\alpha}\right|^{\frac{1}{2}}\right)=2^{\frac{1}{4}} \pi^{\frac{1}{2}} l_{\alpha}^{-\frac{3}{4}},
$$

and denote $a_{\alpha}=\left\|\varphi_{\alpha}\right\|$.

For $z<0$ lying below the bottom of the spectrum of $h_{\alpha}$ we define the following operator in $L_{2}(\mathfrak{N})$ :

$$
W_{\alpha}(z)=\Phi_{\alpha} w_{\alpha}\left(z-\frac{p_{\alpha}^{2}}{2 n_{\alpha}}\right) \Phi_{\alpha}^{*} .
$$

Since $w_{\alpha}\left(z^{\prime}\right)$ is bounded in $L_{2}\left(\mathbb{R}^{3}\right)$ uniformly in $z^{\prime} \leqq z$, the operator $W_{\alpha}(z)$ is bounded in $L_{2}(\mathfrak{N})$. One can verify that similarly to $(2.3)$

$$
W_{\alpha}(z)=I-\left|V_{\alpha}\right|^{\frac{1}{2}} R_{\alpha}(z) V_{\alpha}^{\frac{1}{2}},
$$

where $R_{\alpha}(z)$ is the resolvent of the operator $H_{\alpha}=H_{0}+V_{\alpha}$. Furthermore, analogously to (2.4) we obtain from (3.7) that

$$
W_{\alpha}(z)=\left(I+\left|V_{\alpha}\right|^{\frac{1}{2}} R_{0}(z) V_{\alpha}^{\frac{1}{2}}\right)^{-1} .
$$

In what follows we deal with the operators in various spaces of vector-valued functions $L_{2}^{(3)}(\cdot)$. They will be denoted by bold letters and will be written in the matrix form. We denote by $\operatorname{diag}\left\{T_{1}, T_{2}, T_{3}\right\}$ diagonal matrix-operators with the 
entries $T_{1}, T_{2}, T_{3}$ on the diagonal, where $T_{\alpha}$ are the operators in $L_{2}(\cdot)$. For example the notation $\mathbf{W}(z)=\operatorname{diag}\left\{W_{1}(z), W_{2}(z), W_{3}(z)\right\}$ implies that

$$
\mathbf{W}(z)=\left(\begin{array}{ccc}
W_{1}(z) & 0 & 0 \\
0 & W_{2}(z) & 0 \\
0 & 0 & W_{3}(z)
\end{array}\right)
$$

For a selfadjoint operator $B$ acting in a Hilbert space $\mathfrak{h}$ denote by $\mathfrak{h}_{B}(\lambda) \subset D(B)$, $\lambda \in \mathbb{R}$, a subspace such that $(B f, f)>\lambda\|f\|^{2}$ for any $f \in \mathfrak{h}_{B}(\lambda)$ and set

$$
n(\lambda, B)=\sup _{\mathfrak{h}_{B}(\lambda)} \operatorname{dim} \mathfrak{h}_{B}(\lambda) .
$$

Certainly, for an operator $B$ not having any essential spectrum on the right from the point $\lambda$, the value $n(\lambda, B)$ coincides with the number of eigenvalues of $B$ bigger than $\lambda$. Note the so-called Weyl inequality (see [3]):

$$
n\left(\lambda_{1}+\lambda_{2}, B_{1}+B_{2}\right) \leqq n\left(\lambda_{1}, B_{1}\right)+n\left(\lambda_{2}, B_{2}\right) .
$$

For the operator $H$ we use the following notation: $N(z)=n(-z,-H), z<0$. If inf $\sigma_{\text {ess }}(H)=0$ then $N(z)$ denotes the number of eigenvalues of $H$ on the left from $z$.

The coefficient in the asymptotics of $N(z)$ will be expressed by means of the selfadjoint integral operator $\widehat{\mathbf{S}}(\lambda), \lambda \in \mathbb{R}$, in the space $\mathfrak{G}^{(3)},\left(\mathfrak{5}=L_{2}\left(\mathbb{S}^{2}\right)\right.$ whose kernel depends on the scalar product $t=\langle\xi, \eta\rangle$ of the arguments $\xi, \eta \in \mathbb{S}^{2}$ and has the form

$$
\left.\begin{array}{r}
\hat{S}_{\alpha \alpha}(t ; \lambda)=0, \\
\hat{S}_{\alpha \beta}(t ; \lambda)=(2 \pi)^{-1} u_{\alpha \beta} e^{i r_{\alpha \beta} \lambda} \frac{\sinh \left[\lambda\left(\arccos s_{\alpha \beta} t\right)\right]}{\left(1-s_{\alpha \beta}^{2} t^{2}\right)^{\frac{1}{2}} \sinh (\pi \lambda)},
\end{array}\right\}
$$

where

$$
u_{\alpha \beta}=\kappa_{\alpha \beta}\left(\frac{n_{\alpha} n_{\beta}}{l_{\alpha} l_{\beta}}\right)^{\frac{1}{4}}, \quad r_{\alpha \beta}=\frac{1}{2} \log \frac{l_{\alpha}}{l_{\beta}}, \quad s_{\alpha \beta}=\frac{\left(l_{\alpha} l_{\beta}\right)^{\frac{1}{2}}}{m_{\gamma}},
$$

$\kappa_{\alpha \beta}$ being the number such that $\kappa_{\alpha \beta}=1$ if both subsystems $\alpha$ and $\beta$ have zero energy resonances, otherwise $\kappa_{\alpha \beta}=0$. Because of (3.3) $s_{\alpha \beta}<1$ so that $\arccos s_{\alpha \beta} t<\pi$. Consequently, $\|\hat{\mathbf{S}}(\lambda)\| \rightarrow 0$ as $|\lambda| \rightarrow \infty$. Therefore the integral

$$
\mathfrak{U}(\mu):=(4 \pi)^{-1} \int_{-\infty}^{\infty} n(\mu, \widehat{\mathbf{S}}(\lambda)) d \lambda, \quad \mu>0,
$$

is finite. Denote $\mathfrak{A}_{0}=\mathfrak{U}(1)$. Now we are able to formulate the main result:

Theorem 3.1. Let the pair potentials $v_{\alpha}$ satisfy (2.1) and $v_{\alpha} \leqq 0$. Suppose that $H_{\alpha} \geqq 0$ for all $\alpha$ and that one of the two following conditions is fulfilled:

(1) Zero is the resonance for all two-particle subsystems;

(2) Zero is the resonance for two-particle subsystems $\alpha, \beta$ and is the regular point for the system $\gamma ; m_{\gamma}<\infty$. 
Then the operator $H$ has an infinite negative discrete spectrum and the function $N(z)$ obeys the relation

$$
\lim _{z \rightarrow-0}|\log | z||^{-1} N(z)=\mathfrak{A}_{0},
$$

the r.h.s. of (3.13) being positive.

Clearly, the infinitude of the negative discrete spectrum of $H$ follows automatically from the positivity of $\mathfrak{U}_{0}$. Note that the asymptotics (3.13) does not depend on the potentials $v_{\alpha}$. Furthermore, substituting (3.3) into (3.10) one can check that the operator $\hat{\mathbf{S}}(\lambda)$ and, consequently, the coefficient $\mathfrak{A}_{0}$, depends on the ratios $m_{\alpha} / m_{\beta}$ and $m_{\beta} / m_{\gamma}$ only (for any choice of $\alpha, \beta$ and $\gamma$ ) if $m_{\alpha}<\infty$ for all $\alpha$, and on the ratio $m_{\alpha} / m_{\beta}$ if $m_{\gamma}=\infty$.

Recall that according to our definition given in Sect. 2 the assumption that the value $\lambda=0$ is the resonance for $h_{\alpha}$ suggests that $\lambda=0$ is not an eigenvalue of $h_{\alpha}$. As was pointed out to the author by $\mathrm{H}$. Tamura, the latter condition is automatically fulfilled, if $h_{\alpha}$ has no negative spectrum! In particular, under condition (1) of Theorem 3.1 none of the three two-particle subsystems has eigenvalue $\lambda=0$. Note also that for spherically symmetric pair potentials $v_{\alpha}$ this fact was observed in [13].

The rest of this section is devoted to the detailed discussion of Theorem 3.1, while its proof is postponed until Sect. 4 .

2. It is convenient to calculate the coefficient $\mathfrak{A}_{0}$ by means of decomposition of the operator $\hat{\mathbf{S}}(\lambda)$ into the orthogonal sum over its invariant subspaces. To that end we present $\mathfrak{G}^{(3)}$ as $\mathbb{C}^{3} \otimes \mathfrak{G}$. Denote by $\mathfrak{G}_{l} \subset \mathfrak{G}, l \geqq 0$, the subspace of surface harmonics of degree $l, \sum_{l=0}^{\infty} \oplus \mathfrak{G}_{l}=\mathfrak{G}_{l}, \operatorname{dim} \mathfrak{G}_{l}=2 l+1$. Let $\mathscr{P}_{l}: \mathfrak{G}_{\mathfrak{5}} \rightarrow \mathfrak{G}_{l}$ be the orthogonal projector onto $\mathfrak{G}_{l}$. The kernel of $\mathscr{P}_{l}$ is expressed via the Legendre polynomials $P_{l}(\cdot)$ :

$$
\mathscr{P}_{l}(\xi, \eta)=\frac{2 l+1}{4 \pi} P_{l}(\langle\xi, \eta\rangle)
$$

The kernel of $\widehat{\mathbf{S}}(\lambda)$ depends on the scalar product $\langle\xi, \eta\rangle$ only, so that the subspaces $\mathbb{C}^{3} \otimes \mathfrak{G}_{l}$ are invariant for $\widehat{\mathbf{S}}(\lambda)$ and

$$
\widehat{\mathbf{S}}(\lambda)=\sum_{l=0}^{\infty} \oplus\left(\hat{\mathbf{S}}^{(l)}(\lambda) \otimes \mathscr{P}_{l}\right)
$$

where $\hat{\mathbf{S}}^{(l)}(\lambda)$ are the $3 \times 3$-matrices with the entries

$$
\hat{S}_{\alpha \beta}^{(l)}(\lambda)=2 \pi \int_{-1}^{1} P_{l}(t) \hat{S}_{\alpha \beta}(t ; \lambda) d t
$$

Therefore

$$
n(\mu, \widehat{\mathbf{S}}(\lambda))=\sum_{l=0}^{\infty}(2 l+1) n\left(\mu, \widehat{\mathbf{S}}^{(l)}(\lambda)\right), \quad \mu>0 .
$$

Now, relying on this equality we establish a lower bound for $\mathfrak{A}_{0}$.

Lemma 3.2. If the condition (1) of Theorem 3.1 is fulfilled then the lower bound

$$
\pi^{2} \mathfrak{U}_{0} \geqq \log 2+\frac{1}{3} \log u_{12} u_{23} u_{31}
$$


holds. If the condition (2) of Theorem 3.1 is fulfilled then the lower bound

$$
\pi^{2} \mathfrak{A}_{0} \geqq \log u_{\alpha \beta}
$$

holds. In particular, $\mathfrak{A}_{0}>0$.

Proof. By (3.16) it suffices to prove $\left(3.17^{\prime}\right)$ and (3.17") for the integral

$$
\frac{\pi}{4} \int_{-\infty}^{\infty} n\left(1, \hat{\mathbf{S}}^{(0)}(\lambda)\right) d \lambda
$$

Let us first calculate the entries (3.15):

$$
\hat{S}_{\alpha \beta}^{(0)}(\lambda)=\frac{u_{\alpha \beta} e^{i r_{\alpha \beta} \lambda}}{\sinh (\pi \lambda)} \int_{-1}^{1} \frac{\sinh \left[\lambda\left(\arccos s_{\alpha \beta} t\right)\right]}{\left(1-s_{\alpha \beta}^{2} t^{2}\right)^{\frac{1}{2}}} d t .
$$

The integral here equals

$$
\frac{1}{s_{\alpha \beta}} \int_{\arccos s_{\alpha \beta}}^{\pi-\arccos s_{\alpha \beta}} \sinh \lambda x d x=\frac{2}{s_{\alpha \beta} \lambda} \sinh \frac{\pi \lambda}{2} \sinh \left[\lambda\left(\frac{\pi}{2}-\arccos s_{\alpha \beta}\right)\right] .
$$

Taking into account the identities $\sinh (\pi \lambda)=2 \sinh (\pi \lambda / 2) \cosh (\pi \lambda / 2)$ and $\frac{\pi}{2}-\arccos x=\arcsin x, x \in[0,1]$, we obtain from (3.18):

$$
\hat{S}_{\alpha \beta}^{(0)}(\lambda)=\frac{u_{\alpha \beta} e^{i r_{\alpha \beta} \lambda} \sinh \left(\lambda \arcsin s_{\alpha \beta}\right)}{s_{\alpha \beta} \lambda \cosh \frac{\pi \lambda}{2}} .
$$

Suppose first that the condition (1) of Theorem 3.1 is fulfilled. In this case we use the following simple argument.

Let $S$ be a Hermitian $3 \times 3$-matrix such that $\operatorname{det} S>0$ and $\operatorname{tr} S=0$. Then $S$ has an eigenvalue $\geqq(4 \operatorname{det} S)^{1 / 3}$.

Since

$$
\frac{\sinh \left(\lambda \arcsin s_{\alpha \beta}\right)}{s_{\alpha \beta} \lambda} \geqq 1
$$

we have

$$
\operatorname{det} \hat{\mathbf{S}}^{(0)}(\lambda)=2 \hat{S}_{12}^{(0)}(\lambda) \hat{S}_{23}^{(0)}(\lambda) \hat{S}_{31}^{(0)}(\lambda) \geqq \frac{2 u_{12} u_{13} u_{23}}{\left(\cosh \frac{\pi \lambda}{2}\right)^{3}} .
$$

Therefore, the operator $\widehat{\mathbf{S}}^{(0)}(\lambda)$ has at least one eigenvalue not less than

$$
2\left(u_{12} u_{13} u^{\prime 23}\right)^{\frac{1}{3}} e^{-\frac{\pi|\lambda|}{2}} \text {. }
$$

Thus

$$
\int_{-\infty}^{\infty} n\left(1, \hat{\mathbf{S}}^{(0)}(\lambda)\right) d \lambda \geqq \operatorname{mes}\left\{\lambda \mid 2\left(u_{12} u_{13} u_{23}\right)^{\frac{1}{3}} e^{-\frac{\pi|\lambda|}{2}}>1\right\} .
$$

This gives $\left(3.17^{\prime}\right)$. The positivity of $\mathfrak{A}_{0}$ follows from the fact that $u_{\alpha \beta} \geqq 1$, which is a simple consequence of (3.3) and (3.11). 
Suppose now that the condition (2) of Theorem 3.1 is fulfilled. The matrix $\hat{\mathbf{S}}^{(0)}(\lambda)$ has only two non-trivial entries: $\hat{S}_{\alpha \beta}^{(0)}(\lambda)$ and $\hat{S}_{\beta \alpha}^{(0)}(\lambda)=\widehat{S}_{\alpha \beta}^{(0)}(\lambda)$, so the only positive eigenvalue of $\hat{\mathbf{S}}^{(0)}(\lambda)$ equals $\left|\hat{S}_{\alpha \beta}^{(0)}(\lambda)\right|$. Therefore it has the lower bound

$$
u_{\alpha \beta} e^{-\frac{\pi|\lambda|}{2}} \text {. }
$$

This yields (3.17"). Note that $\mathfrak{A}_{0}>0$ since $u_{\alpha \beta}>1$ under the condition $m_{\gamma}<\infty$. The proof is completed.

3. It should be mentioned that the cases (1) and (2) in Theorem 3.1 are qualitatively different. Namely, according to $\left(3.17^{\prime}\right)$ in case (1) the coefficient $\mathfrak{A}_{0}$ is always separated away from zero for any choice of $m_{1}, m_{2}, m_{3}$;

$$
\mathfrak{A}_{0} \geqq \frac{\log 2}{\pi^{2}} .
$$

On the contrary, the r.h.s. of $\left(3.17^{\prime \prime}\right)$ is positive only for $m_{\gamma}<\infty$. We shall show that in the case when only subsystems $\alpha$ and $\beta$ have zero resonances and $m_{\gamma}=\infty$, the coefficient $\mathfrak{H}_{0}$ equals zero (so that we can not even say that the negative discrete spectrum of $H$ is infinite). Indeed, as in the proof of Lemma 3.2 all the entries of $\hat{\mathbf{S}}(\lambda)$ equal zero except for $\hat{S}_{\alpha \beta}(\lambda)$ and $\hat{S}_{\beta \alpha}(\lambda)=\hat{S}_{\alpha \beta}^{*}(\lambda)$. Evidently

$$
u_{\alpha \beta}=1, \quad r_{\alpha \beta}=\frac{1}{2} \log \frac{m_{\beta}}{m_{\alpha}}, \quad s_{\alpha \beta}=0 .
$$

Thus the kernel of $\hat{S}_{\alpha \beta}(\lambda)$ does not depend on $t$, so that $\hat{\mathbf{S}}^{(l)}(\lambda)=0, l \geqq 1$. According to (3.19) the only positive eigenvalue of $\widehat{\mathbf{S}}(\lambda)$ is $(\cosh \pi \lambda / 2)^{-1}$. It is less than 1 for all $\lambda \neq 0$, so $\mathfrak{A}_{0}=0$.

4. Now we are going to obtain an upper bound for $\mathfrak{A}_{0}$. Since $\left\|\hat{\mathbf{S}}^{(l)}(\lambda)\right\| \rightarrow 0$ as $l \rightarrow \infty$ or $|\lambda| \rightarrow \infty$, in view of (3.16) we have for any $\mu>0$ :

$$
\mathfrak{A}(\mu)=(4 \pi)^{-1} \sum_{l=0}^{L}(2 l+1) \int_{-R}^{R} n\left(\mu, \hat{\mathbf{S}}^{(l)}(\lambda)\right) d \lambda
$$

for $L=L(\mu)<\infty, R=R(\mu)<\infty$ large enough. Together with the inequality $n\left(\mu, \widehat{\mathbf{S}}^{(l)}(\lambda)\right)<3$ this immediately yields:

$$
\mathfrak{A}(\mu) \leqq \frac{3}{2 \pi} R(\mu)(L(\mu)+1)^{2} .
$$

Thus to estimate $\mathfrak{H}_{0}$ from above it suffices to obtain upper bounds for $R(1)$ and $L(1)$. From now on we assume $\mu=1$ and omit $\mu$ from the notations.

First we estimate $L$. Applying the equality

$$
\frac{\sinh \lambda \theta}{\sin \theta \sinh \pi \lambda}=\frac{1}{2 \pi} \int_{-\infty}^{\infty} e^{-i \lambda x} \frac{1}{\cosh x+\cos \theta} d x, \quad 0<\theta<\pi,
$$

established in Appendix I, we see that

$$
\hat{S}_{\alpha \beta}(t ; \lambda)=(2 \pi)^{-2} u_{\alpha \beta} e^{i r_{\alpha \beta} \lambda} \int_{-\infty}^{\infty} e^{-i \lambda x} \frac{1}{\cosh x+S_{\alpha \beta} t} d x .
$$

Recall that (see [5])

$$
\int_{-1}^{1}(z-t)^{-1} P_{l}(t) d t=2 Q_{l}(z), \quad z \notin[-1,1],
$$


where $Q_{l}(z)$ is the Legendre function of the second kind. Taking into account that $Q_{l}(-z)=(-1)^{l+1} Q_{l}(z), z>1$, we obtain from (3.15) that

$$
\begin{aligned}
\hat{S}_{\alpha \beta}^{(l)}(\lambda) & =-\pi^{-1} u_{\alpha \beta} e^{i r_{\alpha \beta} \lambda} s_{\alpha \beta}^{-1} \int_{-\infty}^{\infty} e^{-i \lambda x} Q_{l}\left(-s_{\alpha \beta}^{-1} \cosh x\right) d x \\
& =(-1)^{l} 2 \pi^{-1} u_{\alpha \beta} e^{i r_{\alpha \beta} \lambda} s_{\alpha \beta}^{-1} \int_{0}^{\infty} \cos \lambda x Q_{l}\left(s_{\alpha \beta}^{-1} \cosh x\right) d x .
\end{aligned}
$$

Since (see Appendix II)

$$
\left|Q_{l}(z)\right| \leqq \frac{\pi^{\frac{1}{2}}\left[z+\left(z^{2}-1\right)^{\frac{1}{2}}\right]^{-\left(l+\frac{1}{2}\right)}}{\left(z^{2}-1\right)^{\frac{1}{4}}(2 l+1)^{\frac{1}{2}}}, \quad z>1
$$

we have

$$
\begin{aligned}
\left|\hat{S}_{\alpha \beta}^{(l)}(\lambda)\right| & \leqq 2 \pi^{-1} u_{\alpha \beta} s_{\alpha \beta}^{-1} \int_{1}^{\infty} \frac{\left|Q_{l}\left(s_{\alpha \beta}^{-1} z\right)\right|}{\left(z^{2}-1\right)^{\frac{1}{2}}} d z \\
& \leqq \frac{2 u_{\alpha \beta}}{\pi^{\frac{1}{2}} s_{\alpha \beta}^{\frac{1}{2}}(2 l+1)^{\frac{1}{2}}} \int_{1}^{\infty} \frac{\left[s_{\alpha \beta}^{-1} z+\left(s_{\alpha \beta}^{-2} z^{2}-1\right)^{\frac{1}{2}}\right]^{-\left(l+\frac{1}{2}\right)}}{\left(z^{2}-1\right)^{\frac{1}{2}}\left(z^{2}-s_{\alpha \beta}^{2}\right)^{\frac{1}{4}}} d z .
\end{aligned}
$$

Since $z>1$ we find that

$$
s_{\alpha \beta}^{-1} z+\left(s_{\alpha \beta}^{-2} z^{2}-1\right)^{\frac{1}{2}} \geqq z\left[s_{\alpha \beta}^{-1}+\left(s_{\alpha \beta}^{-2}-1\right)^{\frac{1}{2}}\right] .
$$

Furthermore, using the inequality

$$
\int_{1}^{\infty} \frac{z^{-\left(l+\frac{1}{2}\right)}}{\left(z^{2}-1\right)^{\frac{3}{4}}} d z=\frac{1}{2} \int_{1}^{\infty} \frac{z^{-\left(\frac{1}{2}+\frac{3}{4}\right)}}{(z-1)^{\frac{3}{4}}} d z \leqq \frac{8}{3^{\frac{3}{4}}(2 l+1)^{\frac{1}{4}}},
$$

we arrive at the bound

$$
\left|\hat{S}_{\alpha \beta}^{(l)}(\lambda)\right| \leqq \frac{16 u_{\alpha \beta}\left[s_{\alpha \beta}^{-1}+\left(s_{\alpha \beta}^{-2}-1\right)^{\frac{1}{2}}\right]^{-\left(l+\frac{1}{2}\right)}}{\pi^{\frac{1}{2}} 3^{\frac{3}{4}} S_{\alpha \beta}^{\frac{1}{2}}(2 l+1)^{\frac{3}{4}}} \leqq \frac{16 u_{\alpha \beta}\left[s_{\alpha \beta}^{-1}+\left(s_{\alpha \beta}^{-2}-1\right)^{\frac{1}{2}}\right]^{-l}}{\pi^{\frac{1}{2}} 3^{\frac{3}{4}}(2 l+1)^{\frac{3}{4}}} .
$$

Now, it follows from here and from the obvious inequality

$$
\left\|\hat{\mathbf{S}}^{(l)}(\lambda)\right\| \leqq \max _{\alpha} \sum_{\beta}\left|\hat{S}_{\alpha \beta}^{(l)}(\lambda)\right|,
$$

that

$$
\left\|\hat{\mathbf{S}}^{(l)}(\lambda)\right\| \leqq \frac{16\left[s^{-1}+\left(s^{-2}-1\right)^{\frac{1}{2}}\right]^{-l} u}{\pi^{\frac{1}{2}} 3^{\frac{3}{4}}(2 l+1)^{\frac{3}{4}}}, \quad u:=\max _{\alpha} \sum_{\beta} u_{\alpha \beta}, \quad s:=\max \left\{s_{\alpha \beta}\right\} .
$$

Thus $\left\|\hat{\mathbf{S}}^{(l)}(\lambda)\right\| \leqq 1$ if $l \geqq L$ where

$$
L=\frac{\log \frac{16 u}{\pi^{\frac{1}{2}} 3^{\frac{3}{4}}}}{\log \left[s^{-1}+\left(s^{-2}-1\right)^{\frac{1}{2}}\right]} .
$$


Now we estimate the number $R$ in (3.20). To that end we obtain an upper bound for the norm of the operator $\widehat{\mathbf{S}}(\lambda)$ :

$$
\|\hat{\mathbf{S}}(\lambda)\| \leqq 2 \pi \max _{\alpha} \sum_{\beta} \int_{-1}^{1}\left|\hat{S}_{\alpha \beta}(t ; \lambda)\right| d t=\max _{\alpha} \sum_{\beta}\left|\hat{S}_{\alpha \beta}^{(0)}(\lambda)\right| .
$$

According to (3.19)

$$
\begin{aligned}
\left|\hat{S}_{\alpha \beta}^{(0)}(\lambda)\right| & =\frac{u_{\alpha \beta} \sinh \left(\lambda \arcsin s_{\alpha \beta}\right)}{s_{\alpha \beta} \lambda \cosh \frac{\pi \lambda}{2}} \leqq u_{\alpha \beta} \frac{\arcsin s_{\alpha \beta}}{s_{\alpha \beta}} \frac{\cosh \left(\lambda \arcsin s_{\alpha \beta}\right)}{\cosh \frac{\pi \lambda}{2}} \\
& \leqq \frac{\pi u_{\alpha \beta}}{2} \frac{\cosh \left(\lambda \arcsin s_{\alpha \beta}\right)}{\cosh \frac{\pi \lambda}{2}} \leqq \pi u_{\alpha \beta} e^{|\lambda|\left(\arcsin s_{\alpha \beta}-\frac{\pi}{2}\right)}
\end{aligned}
$$

In view of (3.26) this yields

$$
\|\widehat{\mathbf{S}}(\lambda)\| \leqq \pi u e^{|\lambda|\left(\arcsin s_{\alpha \beta}-\frac{\pi}{2}\right)} .
$$

Therefore $\|\widehat{\mathbf{S}}(\lambda)\| \leqq 1$ if $|\lambda| \geqq R$, where

$$
R=\frac{\log (\pi u)}{\frac{\pi}{2}-\arcsin s}
$$

Putting together (3.20), (3.25) and (3.27) we obtain the upper bound

$$
\begin{gathered}
\mathfrak{U}_{0} \leqq \frac{3}{2 \pi} \frac{\log (\pi u)}{\frac{\pi}{2}-\arcsin s}\left\{\frac{\log \frac{16 u}{\pi^{\frac{1}{2}} 3^{\frac{3}{4}}}}{\log \left[s^{-1}+\left(s^{-2}-1\right)^{\frac{1}{2}}\right]}+1\right\}^{2}, \\
u:=\max _{\alpha} \sum_{\beta} u_{\alpha \beta}, \quad s:=\max \left\{s_{\alpha \beta}\right\} .
\end{gathered}
$$

5. Let us consider the important particular case: $m_{1}=m_{2}=m_{3}$. Now we can calculate the coefficient $\mathfrak{U}_{0}$ explicitly. Let $\lambda_{k}$ be the unique positive solution of the equation

$$
\lambda=2^{4-k} \cdot 3^{-\frac{1}{2}} \frac{\sinh \frac{\pi \lambda}{6}}{\cosh \frac{\pi \lambda}{2}}, \quad k=1,2 .
$$

Then we have

Theorem 3.3. Let $m_{1}=m_{2}=m_{3}$ and one of the conditions (1) or (2) of Theorem 3.1 be fulfilled. Then $\mathfrak{U}_{0}=\lambda_{k} /(2 \pi)$, where $k=1$ for the condition (1) and $k=2$ for the condition (2).

Proof. Note first that $n\left(1, \widehat{\mathbf{S}}^{(l)}(\lambda)\right)=0$ for $l \geqq 1$. Indeed, since

$$
u_{\alpha \beta}=2 \cdot 3^{-\frac{1}{2}} \kappa_{\alpha \beta}, \quad s_{\alpha \beta}=2^{-1}, \quad r_{\alpha \beta}=0,
$$


using the first inequality (3.23), we see that $\left|\hat{S}_{\alpha \beta}^{(l)}(\lambda)\right|<1 / 2$ for $l \geqq 1$. Thus (3.24) yields $\left\|\hat{\mathbf{S}}^{(l)}(\lambda)\right\|<1, l \geqq 1$. Consequently, it remains to study the matrix $\hat{\mathbf{S}}^{(0)}(\lambda)$.

Suppose first that all two-particle subsystems have zero resonances. Then in view of (3.19),

$$
\hat{\mathbf{S}}^{(0)}(\lambda)=a(\lambda) \mathbf{J}_{1}, \quad \mathbf{J}_{1}=\left(\begin{array}{lll}
0 & 1 & 1 \\
1 & 0 & 1 \\
1 & 1 & 0
\end{array}\right),
$$

where

$$
a(\lambda)=4 \cdot 3^{-\frac{1}{2}} \frac{\sinh \frac{\pi \lambda}{6}}{\lambda \cosh \frac{\pi \lambda}{2}} .
$$

The eigenvalues of $\mathbf{J}_{1}$ are $2,-1,-1$, so the only positive eigenvalue of $\hat{\mathbf{S}}^{(0)}(\lambda)$ is $2 a(\lambda)$. Hence

$$
\mathfrak{A}_{0}=\frac{1}{4 \pi} \int n\left(1, \hat{\mathbf{S}}^{(0)}(\lambda)\right) d \lambda=\frac{1}{4 \pi} \int_{2 a(\lambda)>1} d \lambda=\frac{\lambda_{1}}{2 \pi} .
$$

Now suppose that only the subsystems 2 and 3 have zero resonances. Then

$$
\hat{\mathbf{S}}^{(0)}(\lambda)=a(\lambda) \mathbf{J}_{2}, \quad \mathbf{J}_{2}=\left(\begin{array}{lll}
0 & 0 & 0 \\
0 & 0 & 1 \\
0 & 1 & 0
\end{array}\right) .
$$

The eigenvalues of $\mathbf{J}_{2}$ are $-1,0,1$, so the only positive eigenvalue of $\hat{\mathbf{S}}^{(0)}(\lambda)$ is $a(\lambda)$. Hence

$$
\mathfrak{A}_{0}=\frac{1}{4 \pi} \int_{a(\lambda)>1} d \lambda=\frac{\lambda_{2}}{2 \pi} .
$$

The theorem is proven.

Note that the case of identical masses was considered in [7] as well.

6. In conclusion we prove the continuity of $\mathfrak{A}(\mu)$ in $\mu$ which will be necessary when proving Theorem 3.1.

Lemma 3.4. The function $\mathfrak{U}(\mu)$ is continuous in $\mu>0$.

Proof. We need only to prove the continuity of the integral

$$
\int_{-\infty}^{\infty} n\left(\mu, \hat{\mathbf{S}}^{(l)}(\lambda)\right) d \lambda
$$

for fixed $l$. The matrix $\hat{\mathbf{S}}^{(l)}(\lambda)$ is analytic in a neighbourhood of the real axis, so we can numerate its eigenvalue branches $\mu_{k}(\lambda), k=1,2,3$, in such a way that they are analytic as well. In combination with the fact that $\left\|\hat{\mathbf{S}}^{(l)}(\lambda)\right\| \rightarrow 0,|\lambda| \rightarrow \infty$, this implies that the number of points $\lambda_{1}, \lambda_{2}, \ldots, \lambda_{r}$, where at least one of the eigenvalues $\mu_{k}(\lambda)$ coincides with $\mu$, is finite. Clearly, outside of $\lambda_{k}, k=1, \ldots, r$, we have 
$\lim _{\mu^{\prime} \rightarrow \mu} n\left(\mu^{\prime}, \hat{\mathbf{S}}^{(l)}(\lambda)\right)=n\left(\mu, \hat{\mathbf{S}}^{(l)}(\lambda)\right)$. Now the desired continuity follows from the dominated convergence theorem.

\section{Proof of Theorem 3.1}

1. In our analysis of the spectrum of $H$ the crucial role is played by the compact integral operator

$$
\mathbf{A}=\mathbf{A}(z)=(\mathbf{W}(z))^{1 / 2} \mathbf{K}(z)(\mathbf{W}(z))^{1 / 2}
$$

in the space $L_{2}^{(3)}(\mathfrak{N})$, where $\mathbf{K}=\mathbf{K}(z)$ has the entries

$$
\left.\begin{array}{l}
K_{\alpha \alpha}=0, \\
K_{\alpha \beta}=\left|V_{\alpha}\right|^{\frac{1}{2}} R_{0}(z)\left|V_{\beta}\right|^{\frac{1}{2}} .
\end{array}\right\}
$$

The following statement relates the spectra of $H$ and $\mathbf{A}(z)$.

Theorem 4.1. Let $V_{\alpha} \leqq 0$ and $H_{\alpha} \geqq 0$. Then for $z<0$ the operator $\mathbf{A}(z)$ is compact and continuous in $z$ and

$$
N(z)=n(1, \mathbf{A}(z)) .
$$

We start the proof of Theorem 4.1 with two elementary lemmas.

Lemma 4.2. For any bounded operator $B$

$$
n\left(\lambda, B B^{*}\right)=n\left(\lambda, B^{*} B\right) .
$$

Proof. Let us show first that

$$
n\left(\lambda, B^{*} B\right) \leqq n\left(\lambda, B B^{*}\right) .
$$

To that end we present $B$ in the polar form $B=U|B|$, where $|B|^{2}=B^{*} B$ and $U$ is the operator such that $U U^{*}=P, U^{*} U=Q, P$ and $Q$ being the orthogonal projectors onto $\overline{R(B)}$ and $\overline{R\left(B^{*}\right)}$ respectively. Suppose that

$$
\left(|B|^{2} f, f\right)>\lambda\|f\|^{2} \text {. }
$$

Since $|B|^{2}=Q|B|^{2} Q$ one may take $f=Q f$. Therefore $f=U^{*} U f=U^{*} g, g=P g$. Consequently (4.5) yields

$$
\left(U|B|^{2} U^{*} g, g\right)>\lambda\left\|U^{*} g\right\|^{2}=\lambda\|g\|^{2} .
$$

Taking into account that $B B^{*}=U|B|^{2} U^{*}$ we obtain (4.4). To get the opposite inequality it remains to switch $B$ and $B^{*}$.

Let $Z_{j}, j=1,2, \ldots, v, v<\infty$, be a set of bounded operators acting from $\mathfrak{g}$ to $\mathfrak{h}$. Let $M:=\sum_{j=1}^{v} Z_{j}^{*} Z_{j}: \mathfrak{g} \rightarrow \mathfrak{g}$ and

$$
\hat{\mathbf{M}}=\left(\begin{array}{cccc}
Z_{1} Z_{1}^{*} & Z_{1} Z_{2}^{*} & \ldots & Z_{1} Z_{v}^{*} \\
Z_{2} Z_{1}^{*} & Z_{2} Z_{2}^{*} & \ldots & Z_{2} Z_{v}^{*} \\
\vdots & \vdots & \ddots & \vdots \\
Z_{v} Z_{1}^{*} & Z_{v} Z_{2}^{*} & \ldots & Z_{v} Z_{v}^{*}
\end{array}\right)
$$

be the operator in $\sum_{j=1}^{v} \oplus \mathfrak{h}$. 
Lemma 4.3. For any $\lambda>0$

$$
n(\lambda, M)=n(\lambda, \hat{\mathbf{M}})
$$

Proof. Denote by $\mathbf{I}$ the $v \times v$-matrix

$$
\left(\begin{array}{cccc}
1 & 1 & \ldots & 1 \\
1 & 1 & \ldots & 1 \\
\vdots & \vdots & \ddots & \vdots \\
1 & 1 & \ldots & 1
\end{array}\right)
$$

and consider the operator $\mathbf{M}:=M \mathbf{I}$ acting in $\sum_{j=1}^{v} \oplus \mathfrak{g}$. Since $\mathbf{I}$ has a unique non-trivial eigenvalue (which equals $v$ ), we have

$$
n(\lambda, M)=n(\nu \lambda, \mathbf{M}) \text {. }
$$

Direct calculation shows that $\mathbf{M}$ and $\hat{\mathbf{M}}$ have the form $\mathbf{M}=\mathbf{Z} * \mathbf{Z}$ and $\hat{\mathbf{M}}=v^{-1} \mathbf{Z Z} *$ respectively, where

$$
\mathbf{Z}=\left(\begin{array}{cccc}
Z_{1} & Z_{1} & \ldots & Z_{1} \\
Z_{2} & Z_{2} & \ldots & Z_{2} \\
\vdots & \vdots & \ddots & \vdots \\
Z_{v} & Z_{v} & \ldots & Z_{v}
\end{array}\right)
$$

Applying Lemma 4.2 to $\mathbf{Z} * \mathbf{Z}$ and $\mathbf{Z Z}$, we get (4.6) from (4.7).

Proof of the Equality (4.3). First we verify the identity

$$
N(z)=n\left(1, R_{0}^{\frac{1}{2}}(z)|V| R_{0}^{\frac{1}{2}}(z)\right) .
$$

Suppose that $u \in \mathfrak{h}_{-H}(-z)$, i.e. $(H u, u)<z(u, u)$. Then $\left(\left(H_{0}-z\right) u, u\right)<(|V| u, u)$ and consequently,

$$
(y, y)<\left(|V| R_{0}^{\frac{1}{2}}(z) y, R_{0}^{\frac{1}{2}}(z) y\right), \quad y=\left(H_{0}-z\right)^{\frac{1}{2}} u .
$$

Thus $N(z) \leqq n\left(1, R_{0}^{\frac{1}{2}}(z)|V| R_{0}^{\frac{1}{2}}(z)\right)$. Reversing the argument we get the opposite inequality, which proves (4.8).

Since $V=V_{1}+V_{2}+V_{3}$ the operator in the r.h.s. of (4.8) has the form $\sum_{\alpha=1}^{3} Z_{\alpha}^{*} Z_{\alpha}$, where $Z_{\alpha}=\left|V_{\alpha}\right|^{\frac{1}{2}} R_{0}^{\frac{1}{2}}(z)$. Thus by Lemma 4.3 it follows from (4.8) that

$$
N(z)=n(1, \hat{\mathbf{M}}) \text {, }
$$

where $\hat{\mathbf{M}}=\mathbf{M}_{0}+\mathbf{K}, \mathbf{K}$ being defined by (4.2) and $\mathbf{M}_{0}=\operatorname{diag}\left\{Z_{1} Z_{1}^{*}, Z_{2} Z_{2}^{*}, Z_{3} Z_{3}^{*}\right\}$. In view of (3.8) the operator $I-\mathbf{M}_{0}$ is invertible and $\left(I-\mathbf{M}_{0}\right)^{-1}=\mathbf{W}$. Direct calculation shows that

$$
n(1, \hat{\mathbf{M}})=n\left(1, \mathbf{W}^{\frac{1}{2}} \mathbf{K} \mathbf{W}^{\frac{1}{2}}\right) .
$$

The operator in the r.h.s. coincides with $\mathbf{A}(z)$.

To check the compactness and continuity of $\mathbf{A}(z)$ for $z<0$ we first look at a more general operator, whose properties will be useful in what follows. 
Let $\zeta \in C^{\infty}\left(\mathbb{R}_{+}\right)$be the same function as in (2.13). Let $\Gamma_{\alpha}(z)$ be the multiplication by $\zeta\left(\frac{p_{\alpha}^{2}}{2 n_{\alpha}}-z\right)$ and let $\Gamma(z)=\operatorname{diag}\left\{\Gamma_{1}(z), \Gamma_{2}(z), \Gamma_{3}(z)\right\}$. Set

$$
\mathbf{K}^{(\mu, v)}(z)=(\Gamma(z))^{-\mu} \mathbf{K}(z)(\Gamma(z))^{-v} .
$$

Lemma 4.4. Let $v_{\alpha}, \alpha=1,2,3$, satisfy (2.1). Then the operator $\mathbf{K}^{(\mu, v)}(z)$ for $z<0$ is continuous in $z$ and compact for all $v \geqq 0$ and $\mu \geqq 0$. If $v \leqq 1 / 4, \mu \leqq 1 / 4$ and $\mu+v<1 / 2$ then $\mathbf{K}^{(\mu, v)}(z)$ is continuous up to $z=0$.

Proof. Set $\Phi=\operatorname{diag}\left\{\Phi_{1}, \Phi_{2}, \Phi_{3}\right\}$, where $\Phi_{\alpha}$ is the Fourier transform defined in (3.2). It suffices to study the operator

$$
\widetilde{\mathbf{K}}(z)=\widetilde{\mathbf{K}}^{(\mu, v)}(z)=\Phi^{*} \mathbf{K}^{(\mu, v)}(z) \Phi .
$$

The kernel of $\tilde{K}_{\alpha \beta}(z)$ equals

$$
\frac{1}{(2 \pi)^{3}} e^{i d_{z \beta} p x} \frac{\left|v_{\alpha}(x)\right|^{\frac{1}{2}} e^{i e_{\alpha \beta} p^{\prime} x} e^{-i d_{\beta \alpha} p x^{\prime}}\left|v_{\beta}\left(x^{\prime}\right)\right|^{\frac{1}{2}}}{\left[\zeta\left(\frac{p^{2}}{2 n_{\alpha}}-z\right)\right]^{\mu}\left[\zeta\left(\frac{p^{\prime 2}}{2 n_{\beta}}-z\right)\right]^{v}\left[H_{\alpha \beta}^{0}\left(p, p^{\prime}\right)-z\right]} e^{-i e_{\beta x} p^{\prime} x^{\prime}},
$$

where $x=x_{\alpha}, x^{\prime}=x_{\beta}, p=p_{\alpha}, p^{\prime}=p_{\beta}$. Denote by $\xi^{R}$ the multiplication by the characteristic function of the ball $\left\{p \in \mathbb{R}^{3}|| p \mid \leqq R\right\}$. Then, clearly,

$$
\tilde{K}_{\alpha \beta}(z)=Z^{R}(z)+Y^{R}(z),
$$

where

$$
\begin{aligned}
& Z^{R}(z)=\xi^{R} \tilde{K}_{\alpha \beta}(z) \xi^{R}+\left(I-\xi^{R}\right) \tilde{K}_{\alpha \beta}(z) \xi^{R}+\xi^{R} \tilde{K}_{\alpha \beta}(z)\left(I-\xi^{R}\right), \\
& Y^{R}(z)=\left(I-\xi^{R}\right) \tilde{K}_{\alpha \beta}(z)\left(I-\xi^{R}\right) .
\end{aligned}
$$

We shall show that the kernel of the operator (4.10) is square-integrable over its arguments, so that $Z^{R}(z) \in \mathfrak{S}_{2}$. In combination with the continuity of the kernel in $z<0$ this will give the continuity of $Z^{R}(z)$ in $z<0$. Let us consider the first operator in (4.10). For $z<0$ its inclusion in the Hilbert-Schmidt class is obvious. Suppose that $\mu+v<1 / 2, \mu \leqq 1 / 4, v \leqq 1 / 4$ and $z \leqq 0$. In view of (3.5) we have

$$
H_{\alpha \beta}^{0}\left(p, p^{\prime}\right) \geqq c p^{2 \kappa}\left(p^{\prime}\right)^{2 \kappa^{\prime}}, \quad \kappa+\kappa^{\prime}=1 .
$$

Thus the kernel of $Z^{R}(z)$ does not exceed

$$
\left|v_{\alpha}(x)\right|^{\frac{1}{2}} \xi^{R}(p) p^{-2(\mu+\kappa)}\left(p^{\prime}\right)^{-2\left(v+\kappa^{\prime}\right)} \xi^{R}\left(p^{\prime}\right)\left|v_{\beta}\left(x^{\prime}\right)\right|^{\frac{1}{2}} .
$$

It remains to choose $\kappa$ and $\kappa^{\prime}$ in such a way that $\mu+\kappa<3 / 4, v+\kappa^{\prime}<3 / 4$. Using the same argument one can prove that the second and the third operators in (4.10) belong to the Hilbert-Schmidt class and are continuous in $z \leqq 0$ if $\mu \leqq 1 / 4, v \leqq 1 / 4$ and $\mu+v<1 / 2$. For example, the second operator is Hilbert-Schmidt since its kernel is bounded by

$$
C\left|v_{\alpha}(x)\right|^{\frac{1}{2}}\left[1-\xi^{R}(p)\right] p^{-2}\left(\frac{p^{\prime 2}}{2 n_{\beta}}-z\right)^{-v} \xi^{R}\left(p^{\prime}\right)\left|v_{\beta}\left(x^{\prime}\right)\right|^{\frac{1}{2}} .
$$

The norm of the operator (4.11) is bounded by $C R^{-2}$ for all $z \leqq 0$ since

$$
\xi^{R}(p)\left[H_{\alpha \beta}^{0}\left(p, p^{\prime}\right)-z\right] \xi^{R}\left(p^{\prime}\right) \geqq c R^{2}
$$


by (3.5). Therefore $\left\|Z^{R}(z)-\tilde{K}_{\alpha \beta}(z)\right\| \rightarrow 0$ as $R \rightarrow \infty$. Hence the continuity and compactness of $\tilde{K}_{\alpha \beta}(z)$ (and consequently, $K_{\alpha \beta}^{(\mu, v)}(z)$ ) follow from those of $Z^{R}(z)$.

The end of the proof of Theorem 4.1. Comparing (4.1) and (4.9) we see that $\mathbf{A}(z)=\mathbf{W}^{\frac{1}{2}} \mathbf{K}^{(0,0)}(z) \mathbf{W}^{\frac{1}{2}}$. The desired compactness and continuity follow from Lemma 4.4.

2. As we shall see later, the discrete asymptotics of the operator $\mathbf{A}(z)$ as $z \rightarrow-0$ is determined by the integral operator $\mathbf{S}_{R}$ in $L_{2}\left((0, R), \mathfrak{5}^{(3)}\right),\left(\mathfrak{5}=L_{2}\left(\mathbb{S}^{2}\right)\right.$, with the kernel $S_{\alpha \beta}\left(x-x^{\prime} ;\langle\xi, \eta\rangle\right), \eta, \xi \in \mathbb{S}^{2}$, where

and $u_{\alpha \beta}, r_{\alpha \beta}, s_{\alpha \beta}$ are defined in (3.11).

$$
\left.\begin{array}{l}
S_{\alpha \alpha}(x ; t)=0, \\
S_{\alpha \beta}(x ; t)=(2 \pi)^{-2} \frac{u_{\alpha \beta}}{\cosh \left(x+r_{\alpha \beta}\right)+s_{\alpha \beta} t},
\end{array}\right\}
$$

Let the function $\mathfrak{A}(\cdot)$ be defined by (3.12). First of all we establish the following Theorem 4.5. Let $\mathbf{S}_{\boldsymbol{R}}$ be the operator defined in (4.13). Then for any $\mu>0$,

$$
\lim _{R \rightarrow \infty} R^{-1} n\left(\mu, \mathbf{S}_{R}\right)=2 \mathfrak{U}(\mu) .
$$

The proof of Theorem 4.5 is based on a simple Toeplitz type argument. Namely, let us consider the selfadjoint integral operator $\mathbf{T}_{R}$ in the space $L_{2}^{(d)}(0, R), d \geqq 1$, with the kernel $T_{\alpha \beta}\left(x-x^{\prime}\right)$,

$$
T_{\alpha \beta}(x)=\bar{T}_{\beta \alpha}(-x), \quad T_{\alpha \beta} \in L_{1}(\mathbb{R}) \cap L_{\infty}(\mathbb{R}) .
$$

Let $\hat{\mathbf{T}}(\lambda)=\left\{\hat{T}_{\alpha \beta}(\lambda\}\right)$ be the matrix with the entries

$$
\hat{T}_{\alpha \beta}(\lambda)=\int_{-\infty}^{\infty} T_{\alpha \beta}(x) e^{-i \lambda x} d x .
$$

Let $\mu_{k}(\lambda)$ be the eigenvalues of the matrix $\widehat{\mathbf{T}}(\lambda)$ numerated in non-increasing order counting multiplicity. Clearly, they are continuous in $\lambda$. Denote $\mathfrak{M}(\mu)=\mathfrak{M}(\mu ; \widehat{\mathbf{T}})$ $=\bigcup_{k}\left\{\lambda: \mu_{k}(\lambda)=\mu\right\}$.

Lemma 4.6. Let $\mathbf{T}_{R}$ be as defined above and $\mu>0$ be fixed. Suppose that mes $\mathfrak{M}(\mu)=0$. Then we have

$$
2 \pi \lim _{\boldsymbol{R} \rightarrow \infty} R^{-1} n\left(\mu, \mathbf{T}_{R}\right)=\int_{-\infty}^{\infty} n(\mu, \widehat{\mathbf{T}}(\lambda)) d \lambda .
$$

This lemma is a trivial generalization of a corresponding result for the case $d=1$, which can be found in [8]. In the language of Toeplitz operators it provides the so-called canonical distribution for the kernel $T_{\alpha \beta}(x)$. Below we denote by $\|K\|_{2}$ the Hilbert-Schmidt norm of the operator $K$.

Proof of Lemma 4.6. By Riemann-Lebesgue Theorem $n(\mu, \widehat{\mathbf{T}}(\lambda))=0$ for $|\lambda| \geqq M$ with $M>0$ large enough, so that the equality (4.15) is equivalent to

$$
2 \pi \lim _{R \rightarrow \infty} R^{-1} n\left(\mu, \mathbf{T}_{R}\right)=\int_{-M}^{M} n(\mu, \hat{\mathbf{T}}(\lambda)) d \lambda .
$$


Let us define in $L_{2}^{(d)}(0, R)$ the operator $\mathbf{T}_{A, R}$ with the kernel

$$
T_{\alpha \beta}(x ; A)=T_{\alpha \beta}(x) \chi(x / A), \quad A \leqq R / 2,
$$

where $\chi$ means the characteristic function of the interval $[-1,1]$. Then, obviously,

$$
\left\|\mathbf{T}_{R}-\mathbf{T}_{A, R}\right\|_{2}^{2} \leqq R \sum_{\alpha \beta} \int_{|x| \geqq A}\left|T_{\alpha \beta}(x)\right|^{2} d x
$$

Further, let

$$
\mathscr{T}_{\alpha \beta}(x ; A)=\sum_{n=-\infty}^{\infty} T_{\alpha \beta}(x+R n ; A)
$$

be a periodic extension of the kernel $T_{\beta}(x ; A)$ with period $R$. Denote by $\mathbf{T}_{A, R}^{p}$ the operator in $L_{2}^{(d)}(0, R)$ with the kernel (4.18) and estimate the Hilbert-Schmidt norm

$$
\left\|\mathbf{T}_{A, R}-\mathbf{T}_{A, R}^{p}\right\|_{2}^{2}=\sum_{\alpha \beta} \int_{0}^{R} d x^{\prime} \int_{0}^{R} d x\left|\sum_{n \neq 0} T_{\alpha \beta}\left(x-x^{\prime}+R n ; A\right)\right|^{2} .
$$

In fact, the r.h.s. contains only summands with $n=-1, n=-1$. All the others equal zero for $x, x^{\prime} \in[0, R]$. Therefore

$$
\begin{aligned}
\left\|\mathbf{T}_{A, R}-\mathbf{T}_{A, R}^{p}\right\|_{2}^{2} \leqq & 2 \sum_{\alpha \beta} \int_{0}^{R} d x^{\prime} \int_{0}^{R} d x\left\{\left|T_{\alpha \beta}\left(x-x^{\prime}+R\right)\right|^{2}\right. \\
& \left.+\left|T_{\alpha \beta}\left(x-x^{\prime}-R\right)\right|^{2}\right\} \\
\leqq & 2 \sum_{\alpha \beta}\left\{\int_{R-A}^{R} d x^{\prime} \int_{R-x^{\prime}}^{2 R-x^{\prime}} d x\left|T_{\alpha \beta}(x)\right|^{2}\right. \\
& \left.+\int_{0}^{A} d x^{\prime} \int_{-R-x^{\prime}}^{-x^{\prime}} d x\left|T_{\alpha \beta}(x)\right|^{2}\right\} \\
\leqq & 4 A \sum_{\alpha \beta}\left\|T_{\alpha \beta}\right\|_{L_{2}}^{2} .
\end{aligned}
$$

Combining this estimate with (4.17) we obtain

$$
\begin{gathered}
\left\|\mathbf{T}_{R}-\mathbf{T}_{A, R}^{p}\right\|_{2}^{2} \leqq R \kappa(A)+C A, \\
\lim _{A \rightarrow \infty} \kappa(A)=0 .
\end{gathered}
$$

Now it follows from the inequality $n(s, A) \leqq s^{-2}\|A\|_{2}^{2}, s>0$, and the Weyl inequality (3.9) that

$$
\begin{aligned}
n\left(\mu, \mathbf{T}_{R}\right) & \leqq n\left(\mu-\delta, \mathbf{T}_{A, R}^{p}\right)+n\left(\delta, \mathbf{T}_{R}-\mathbf{T}_{A, R}^{p}\right) \\
& \leqq n\left(\mu-\delta, \mathbf{T}_{A, R}^{p}\right)+\delta^{-2}(R \kappa(A)+C A), \\
n\left(\mu, \mathbf{T}_{R}\right) & \geqq n\left(\mu+\delta, \mathbf{T}_{A, R}^{p}\right)-\delta^{-2}(R \kappa(A)+C A),
\end{aligned}
$$


for any $\mu>0, \delta>0$. Consequently,

$$
\left.\begin{array}{c}
\underset{R \rightarrow \infty}{\limsup } R^{-1} n\left(\mu, \mathbf{T}_{R}\right) \leqq \lim \sup R^{-1} n\left(\mu-\delta, \mathbf{T}_{A, R}^{p}\right), \\
\underset{R \rightarrow \infty}{\liminf R^{-1} n\left(\mu, \mathbf{T}_{R}\right) \geqq \liminf R^{-1} n\left(\mu+\delta, \mathbf{T}_{A, R}^{p}\right),}
\end{array}\right\} \forall \delta>0,
$$

where limits in the r.h.s. are taken as $R \rightarrow \infty, A \rightarrow \infty, A / R \rightarrow 0$.

We shall prove that for any $\varepsilon>0$ and some $\delta=\delta(\varepsilon)$, the relation holds:

$$
2 \pi \lim \sup R^{-1} n\left(v, \mathbf{T}_{A, R}^{p}\right) \leqq \int_{-M}^{M} n(\mu, \hat{\mathbf{T}}(\lambda)) d \lambda+C \varepsilon, \quad v=\mu-\delta .
$$

The eigenvalues of the operator $\mathbf{T}_{A, \boldsymbol{R}}^{p}$ can be calculated by means of the Fourier series:

$$
n\left(v, \mathbf{T}_{A, R}^{p}\right)=\sum_{m} n\left(v, \hat{\mathbf{T}}_{A, R}^{(m)}\right),
$$

where $\widehat{\mathbf{T}}_{A, R}^{(m)}$ is the matrix with entries

$$
\int_{-A}^{A} T_{\alpha \beta}(x) e^{-i 2 \frac{m}{R} \pi x} d x .
$$

By condition of lemma for any $\varepsilon>0$ one can find an open set $\mathfrak{M}_{\varepsilon}(\mu)$ (which is a union of a finite number of open intervals) such that

$$
\mathfrak{M}(\mu) \subset \mathfrak{M}_{\varepsilon}(\mu), \quad \operatorname{mes} \mathfrak{M}_{\varepsilon}(\mu)<\varepsilon .
$$

Let us define the number $\delta=\delta(\varepsilon)$ in (4.20) by the requirement

$$
\left|\mu_{k}(\lambda)-\mu\right| \geqq 2 \delta, \quad \forall \lambda \notin \mathfrak{M}_{\varepsilon}(\mu) .
$$

We split the sum in (4.21) into two parts:

$$
\sum_{m} n\left(v, \hat{\mathbf{T}}_{A, R}^{(m)}\right)=\sum_{\frac{2 m \pi}{R} \in \mathfrak{M}_{\ell}(\mu)} n\left(v, \widehat{\mathbf{T}}_{A, R}^{(m)}\right)+\sum_{\frac{2 m \pi}{R} \notin \mathfrak{M}_{\ell}(\mu)} n\left(v, \widehat{\mathbf{T}}_{A, R}^{(m)}\right) .
$$

Since the first term in the r.h.s. is bounded by $R d(2 \pi)^{-1}$ mes $\mathfrak{M}_{\varepsilon}(\mu)$, its contribution to the asymptotics (4.20) does not exceed $C \varepsilon$. Further, according to (4.22) we have

$$
\left\|\hat{\mathbf{T}}_{A, R}^{(m)}-\hat{\mathbf{T}}\left(\frac{2 m}{R} \pi\right)\right\| \rightarrow 0, \quad A \rightarrow \infty .
$$

Since $v=\mu-\delta$, in combination with (4.23) this tells us that $n\left(v, \hat{\mathbf{T}}_{A, R}^{(m)}\right)=$ $n(\mu, \widehat{\mathbf{T}}(2 m \pi / R))$ for large $A$ if $2 m \pi / R \notin \mathfrak{M}_{\varepsilon}(\mu)$. Therefore the second sum in the r.h.s. of (4.24) equals

$$
\frac{R}{2 \pi} \sum_{\frac{2 m \pi}{R} \in[-M, M] \backslash \mathfrak{M}_{\varepsilon}(\mu)} \frac{2 \pi}{R} n\left(\mu, \hat{\mathbf{T}}\left(\frac{2 m}{R} \pi\right)\right),
$$


where $M$ is the same as in (4.16). Since the function $n(\mu, \widehat{\mathbf{T}}(\lambda))$ is continuous in $\lambda \notin \mathfrak{M}_{\varepsilon}(\mu)$ the sum (4.25) considered as the integral sum, converges to the integral

$$
\int_{[-M, M] \backslash \mathbb{M}_{t}(\mu)} n(\mu, \hat{\mathbf{T}}(\lambda)) d \lambda .
$$

To obtain the relation (4.20) it remains to recall once again that mes $\mathfrak{M}_{\varepsilon}(\mu)<\varepsilon$.

Since $\varepsilon>0$ is an arbitrary number, the inequalities (4.19) and (4.20) yield:

$$
2 \pi \limsup _{R \rightarrow \infty} R^{-1} n\left(\mu, \mathbf{T}_{R}\right) \leqq \int_{-M}^{M} n(\mu, \hat{\mathbf{T}}(\lambda)) d \lambda .
$$

In the same way one can establish a similar lower bound for $\lim \inf R^{-1} n\left(\mu, \mathbf{T}_{R}\right)$. Together they prove (4.16).

Proof of the Equality (4.14). Similarly to (3.14) we have

$$
\mathbf{S}_{R}=\sum_{l=0}^{\infty} \oplus\left(\mathbf{S}_{R}^{(l)} \otimes \mathscr{P}_{l}\right)
$$

where $\mathbf{S}_{R}^{(l)}$ are the operators in $L_{2}^{(3)}(0, R)$ with the kernels

$$
S_{\alpha \beta}^{(l)}\left(x-x^{\prime}\right)=2 \pi \int_{-1}^{1} P_{l}(t) S_{\alpha \beta}\left(x-x^{\prime} ; t\right) d t .
$$

Consequently,

$$
n\left(\mu, \mathbf{S}_{R}\right)=\sum_{l=0}^{\infty}(2 l+1) n\left(\mu, \mathbf{S}_{R}^{(l)}\right) .
$$

Now, comparing the definitions (3.10), (4.13) and applying the equality (3.21), we see that

$$
\hat{S}_{\alpha \beta}(t ; \lambda)=\int_{-\infty}^{\infty} e^{-i \lambda x} S_{\alpha \beta}(x ; t) d x .
$$

By (3.15) and (4.26) this yields

$$
\hat{S}_{\alpha \beta}^{(l)}(\lambda)=\int_{-\infty}^{\infty} e^{-i \lambda x} S_{\alpha \beta}^{(l)}(x) d x .
$$

As it was mentioned in the proof of Lemma 3.4, the matrix $\widehat{\mathbf{S}}^{(l)}(\lambda)$ is analytic in $\lambda$ in a neighbourhood of the real axes, so that $\operatorname{mes} \mathfrak{M}\left(\mu ; \hat{\mathbf{S}}^{(l)}\right)=0$ for any $\mu>0$. Applying Lemma 4.6 we find that

$$
2 \pi \lim _{R \rightarrow \infty} R^{-1} n\left(\mu, \mathbf{S}_{R}^{(l)}\right)=\int_{-\infty}^{\infty} n\left(\mu, \hat{\mathbf{S}}^{(l)}(\lambda)\right) d \lambda .
$$

It remains to take into account (4.27) and (3.16).

3. Let us proceed to the study of the operator $\mathbf{A}(z)$. Below we always assume $z \leqq 0$. Our aim is to prove

Theorem 4.7. Let the conditions of Theorem 3.1 be fulfilled. Then for any $\mu>0$ we have

$$
\lim _{z \rightarrow-0}|\log | z \|^{-1} n(\mu, \mathbf{A}(z))=\mathfrak{A}(\mu) .
$$

When proving this theorem we rely upon the following two lemmas. 
Lemma 4.8. Let $T_{1}, T_{2}$ be bounded operators. If $\lambda \neq 0$ is an eigenvalue of $T_{1} T_{2}$ then $\lambda$ is an eigenvalue for $T_{2} T_{1}$ as well of the same algebraic and geometric multiplicities.

This lemma is well known and its proof is omitted.

Lemma 4.9. Let $T(z)=T_{0}(z)+T_{1}(z)$, where $T_{0}\left(T_{1}\right)$ is compact and continuous in $z<0(z \leqq 0)$. Assume that for some function $f(\cdot), f(z) \rightarrow 0, z \rightarrow-0$ there exists the limit

$$
\lim _{z \rightarrow-0} f(z) n\left(\lambda, T_{0}(z)\right)=l(\lambda)
$$

continuous in $\lambda>0$. Then the same limit exists for $T(z)$ and

$$
\lim _{z \rightarrow-0} f(z) n(\lambda, T(z))=l(\lambda) .
$$

Proof. According to the Weyl inequality (3.9) for any $\varepsilon \in(0,1)$ we have

$$
\begin{gathered}
n(\lambda, T(z)) \leqq n\left(\lambda(1-\varepsilon), T_{0}(z)\right)+n\left(\lambda \varepsilon, T_{1}(z)\right), \\
n(\lambda, T(z)) \geqq n\left(\lambda(1+\varepsilon), T_{0}(z)\right)-n\left(\lambda \varepsilon,-T_{1}(z)\right) .
\end{gathered}
$$

Since $T_{1}(z)$ is continuous up to $z=0$, we have

$$
l(\lambda(1+\varepsilon)) \leqq \liminf _{z \rightarrow-0} f(z) n(\lambda, T(z)) \leqq \limsup _{z \rightarrow-0} f(z) n(\lambda, T(z)) \leqq l(\lambda(1-\varepsilon)) .
$$

Now the desired result follows from the continuity of $l(\cdot)$.

Proof of Theorem 4.7. Since $\mathfrak{A}(\cdot)$ is continuous in $\mu$ (see Lemma 3.4), according to Lemma 4.9 the perturbations of the operator $A(z)$ which are compact and continuous up to $z=0$, do not contribute to the asymptotics (4.28). We shall use this fact throughout the proof without further comments.

First we prove the theorem under the condition that all two particle subsystems have zero energy resonances.

Let $\Gamma_{\alpha}(z)$ be the multiplication by $\zeta\left(\frac{p_{\alpha}^{2}}{2 n_{\alpha}}-z\right)$ and $\Pi_{\alpha}$ be the operator such that

$$
\left(\Pi_{\alpha} f\right)\left(k_{\alpha}, p_{\alpha}\right)=a_{\alpha}^{-1}\left(\Phi_{\alpha} \varphi_{\alpha}\right)\left(k_{\alpha}\right) \int f\left(k_{\alpha}^{\prime}, p_{\alpha}\right) \overline{\left(\Phi_{\alpha} \varphi_{\alpha}\right)\left(k_{\alpha}^{\prime}\right)} d k_{\alpha}^{\prime} .
$$

It follows from (2.13) and from the definition of $W_{\alpha}(z)$ that

$$
\begin{aligned}
\left(W_{\alpha}(z)\right)^{\frac{1}{2}} & =\left(\Gamma_{\alpha}(z)\right)^{-\frac{1}{4}} \Pi_{\alpha}+\left(\Gamma_{\alpha}(z)\right)^{-\frac{1-\delta}{4}} \tilde{W}_{\alpha}^{(\delta)}(z) \\
& =\Pi_{\alpha}\left(\Gamma_{\alpha}(z)\right)^{-\frac{1}{4}}+\tilde{W}_{\alpha}^{(\delta)}(z)\left(\Gamma_{\alpha}(z)\right)^{-\frac{1-\delta}{4}},
\end{aligned}
$$

where

$$
\tilde{W}_{\alpha}^{(\delta)}(z)=\Phi_{\alpha} \tilde{w}_{\alpha}^{(\delta)}\left(z-\frac{p_{\alpha}^{2}}{2 n_{\alpha}}\right) \Phi_{\alpha}^{*}
$$

is bounded and continuous in $z \leqq 0$. Thus

$$
\begin{aligned}
(\mathbf{W}(z))^{\frac{1}{2}} & =(\Gamma(z))^{-\frac{1}{4}} \Pi+(\Gamma(z))^{-\frac{1-\delta}{4}} \tilde{\mathbf{W}}^{(\delta)}(z) \\
& =\Pi(\Gamma(z))^{-\frac{1}{4}}+\tilde{\mathbf{W}}^{(\delta)}(z)(\Gamma(z))^{-\frac{1-\delta}{4}},
\end{aligned}
$$


where $\Gamma(z), \tilde{\mathbf{W}}^{(\delta)}(z), \Pi$ are diagonal matrices with the entries $\Gamma_{\alpha}(z), \tilde{W}_{\alpha}^{(\delta)}(z), \Pi_{\alpha}$. It follows from here that $\mathbf{A}(z)=\mathbf{A}^{0}(z)+\mathbf{Y}(z)$, where

$$
\begin{aligned}
\mathbf{A}^{0}(z) & =\Pi \mathbf{K}^{\left(\frac{1}{4}, \frac{1}{4}\right)}(z) \Pi, \\
\mathbf{Y}(z) & =\Pi \mathbf{K}^{(\mu, v)}(z) \tilde{\mathbf{W}}^{(\delta)}(z)+\tilde{\mathbf{W}}^{(\delta)}(z) \mathbf{K}^{(v, \mu)}(z) \Pi+\tilde{\mathbf{W}}(\delta)(z) \mathbf{K}^{(v, v)}(z) \tilde{\mathbf{W}}^{(\delta)}(z) .
\end{aligned}
$$

Here $\mu=1 / 4, v=(1-\delta) / 4$. By Lemma 4.4 the operators $\mathbf{K}^{(\mu, v)}(z), \mathbf{K}^{(v, \mu)}(z)$, $\mathbf{K}^{(v, v)}(z)$ are compact and continuous in $z \leqq 0$. Thus, by virtue of Lemma $4.9 \mathbf{Y}(z)$ does not contribute to the asymptotics (4.28).

Let us look at the operator $\mathbf{A}^{0}(z)$. Let $\xi(\cdot)$ be a characteristic function of the ball $\{p|| p \mid \leqq 1\}$, and $\xi_{\alpha}$ be a multiplication by the function $\xi\left(p_{\alpha}\right)$. Set $\Xi:=$ $\operatorname{diag}\left\{\xi_{1}, \xi_{2}, \xi_{3}\right\}$. Using Lemma 3.6 one can easily prove that $\mathbf{A}^{0}(z)-\Xi \mathbf{A}^{0}(z) \Xi$ is compact and continuous in $z$ up to $z=0$.

The following step consists in a reduction of the problem to an operator acting in the space $L_{2}^{(3)}\left(\mathbb{R}^{3}\right)$. Let $\mathbf{F}=\operatorname{diag}\left\{F_{1}, F_{2}, F_{3}\right\}: L_{2}^{(3)}\left(\mathbb{R}^{3}\right) \rightarrow L_{2}^{(3)}(\mathfrak{N})$ be the operator with the entries

$$
\left(F_{\alpha} f\right)\left(k_{\alpha}, p_{\alpha}\right)=\left(\Phi_{\alpha} \varphi_{\alpha}\right)\left(k_{\alpha}\right) f\left(p_{\alpha}\right)
$$

Then obviously

$$
\left(F_{\alpha}^{*} f\right)\left(p_{\alpha}\right)=\int \overline{\left(\Phi_{\alpha} \varphi_{\alpha}\right)\left(k_{\alpha}\right)} f\left(k_{\alpha}, p_{\alpha}\right) d k_{\alpha} .
$$

The nontrivial eigenvalues of $\Xi \mathbf{A}^{0}(z) \Xi$ coincide with those of the operator

$$
\mathbf{S}(z):=\mathbf{F}^{*} \Xi \mathbf{K}^{\left(\frac{1}{4}, \frac{1}{4}\right)}(z) \Xi \mathbf{F} .
$$

Indeed, by Lemma 4.8 the discrete spectrum of $\Xi \mathbf{A}^{0}(z) \Xi$ coincides with that of

$$
\Xi \mathbf{K}^{\left(\frac{1}{4}, \frac{1}{4}\right)}(z) \Xi \Pi^{2} .
$$

Since $\Pi_{\alpha}^{2}=\left(\cdot, \Phi_{\alpha} \varphi_{\alpha}\right) \Phi_{\alpha} \varphi_{\alpha}=F_{\alpha} F_{\alpha}^{*}$ it remains to apply Lemma 4.8 once again.

One may think that the operator $\mathbf{S}(z)$ acts in $L_{2}^{(3)}\left(B_{1}\right), B_{r}=\{|p| \leqq r\}, r>0$. Its kernel equals

$$
\frac{\overline{\psi_{\alpha}\left(d_{\alpha \beta} p+e_{\alpha \beta} q\right)} \psi_{\beta}\left(d_{\beta \alpha} q+e_{\beta \alpha} p\right)}{\zeta\left(\frac{p^{2}}{2 n_{\alpha}}-z\right)^{\frac{1}{4}}\left(H_{\alpha \beta}^{0}(p, q)-z\right) \zeta\left(\frac{q^{2}}{2 n_{\beta}}-z\right)^{\frac{1}{4}}},
$$

where $\psi_{\alpha}(k)=\left(\Phi_{\alpha}\left|v_{\alpha}\right|^{\frac{1}{2}} \varphi_{\alpha}\right)(k)$. First we replace the functions $\psi_{\alpha}(k)$ by $\psi_{\alpha}(0)=2^{-5 / 4} \pi^{-1} l_{\alpha}^{-3 / 4}$ (see (3.6)). Then by (2.10) and (4.12) the kernel of the difference will be bounded by

$$
\begin{aligned}
C \frac{|p|^{\delta}+|q|^{\delta}}{\left(\frac{p^{2}}{2 n_{\alpha}}-z\right)^{\frac{1}{4}}\left(H_{\alpha \beta}^{0}(p, q)-z\right)\left(\frac{q^{2}}{2 n_{\beta}}-z\right)^{\frac{1}{4}} \leqq} & C^{\prime}|p|^{\delta-2 \kappa-\frac{1}{2}}|q|^{-2 \kappa^{\prime}-\frac{1}{2}} \\
& +C^{\prime}|p|^{-2 \kappa^{\prime}-\frac{1}{2}}|q|^{\delta-2 \kappa-\frac{1}{2}}
\end{aligned}
$$

for arbitrary $\kappa, \kappa^{\prime}, \kappa+\kappa^{\prime}=1$. Choosing $\kappa \in(1 / 2,(1+\delta) / 2)$, we see that this operator is Hilbert-Schmidt up to $z=0$. For the same reason we can replace the functions $\zeta\left(p^{2} / 2 n_{\alpha}-z\right), \zeta\left(q^{2} / 2 n_{\beta}-z\right)$ by $p^{2} / 2 n_{\alpha}-z, q^{2} / 2 n_{\beta}-z$ respectively. Thus 
we arrive at the operator with the following kernel:

$$
2^{-\frac{5}{2}} \pi^{-2}\left(l_{\alpha} l_{\beta}\right)^{-\frac{3}{4}}\left(\frac{p^{2}}{2 n_{\alpha}}-z\right)^{-\frac{1}{4}}\left(\frac{q^{2}}{2 n_{\beta}}-z\right)^{-\frac{1}{4}}\left(H_{\alpha \beta}^{0}(p, q)-z\right)^{-1} .
$$

One can trivially verify that this operator is unitarily equivalent to that with the kernel

$$
2^{-\frac{5}{2}} \pi^{-2}\left(l_{\alpha} l_{\beta}\right)^{-\frac{3}{4}}\left(\frac{p^{2}}{2 n_{\alpha}}+1\right)^{-\frac{1}{4}}\left(\frac{q^{2}}{2 n_{\beta}}+1\right)^{-\frac{1}{4}}\left(H_{\alpha \beta}^{0}(p, q)+1\right)^{-1},
$$

acting in $L_{2}^{(3)}\left(B_{r}\right), r=|z|^{-1 / 2}$. The equivalence is performed by the unitary dilation $\mathbf{U}_{r}=\operatorname{diag}\left\{U_{r}, U_{r}, U_{r}\right\}: L_{2}^{(3)}\left(B_{1}\right) \rightarrow L_{2}^{(3)}\left(B_{r}\right), \quad\left(U_{r} f\right)(p)=r^{-3 / 2} f\left(r^{-1} p\right)$. Further, we may replace $\left(p^{2} / 2 n_{\alpha}+1\right)^{-1},\left(q^{2} / 2 n_{\beta}+1\right)^{-1}$ and $H_{\alpha \beta}^{0}(p, q)+1$ by $2 n_{\alpha} p^{-2}(1-\xi(p)), 2 n_{\beta} q^{-2}(1-\xi(q))$ and $H_{\alpha \beta}^{0}(p, q)$ respectively, since the error will be a Hilbert-Schmidt operator continuous up to $z=0$. Then we get the operator in $L_{2}^{(3)}\left(B_{r} \backslash B_{1}\right)$ with the kernel

$$
(2 \pi)^{-2}\left(n_{\alpha} n_{\beta}\right)^{\frac{1}{4}}\left(l_{\alpha} l_{\beta}\right)^{-\frac{3}{4}}|p|^{-\frac{1}{2}}|q|^{-\frac{1}{2}}\left(\frac{p^{2}}{2 l_{\beta}}+\frac{\langle p, q\rangle}{m_{\gamma}}+\frac{q^{2}}{2 l_{\alpha}}\right)^{-1} .
$$

Here we used the formula (3.4) for $H_{\alpha \beta}^{0}(p, q)$. Finally, this operator is unitarily equivalent to the operator $S_{R}, R=1 / 2|\log | z||$ defined in (4.13). The equivalence is performed by the unitary operator $\mathbf{M}=\operatorname{diag}\{M, M, M\}: L_{2}^{(3)}\left(B_{r} \backslash B_{1}\right) \rightarrow$ $L_{2}\left((0, R), \tilde{5}^{(3)}\right)$, where $(M f)(x, \omega)=e^{3 x / 2} f\left(e^{x} \omega\right), x \in(0, R), \omega \in \mathbb{S}^{2}$. Now the relation (4.28) follows from (4.14).

Now suppose that condition (2) of Theorem 3.1. is fulfilled. To be definite we assume that zero is the regular point for the Hamiltonian $h_{3}$ and the resonance for $h_{1}, h_{2}$. Then by Lemma 2.2 the operator $W_{3}(z) \geqq 0$ is continuous in $z \leqq 0$. Thus setting $\varphi_{3}=0$ we see that $W_{3}(z)$ satisfies the relation (4.25) where

$$
\tilde{W}_{3}^{(\delta)}(z)=\left(\Gamma_{3}(z)\right)^{\frac{1-\delta}{4}} W_{3}(z)
$$

After this remark the proof goes as before.

Now the equality (3.13) follows from Theorem 4.7 and the relation (4.3). This completes the proof of Theorem 3.1.

\section{Appendix}

I. Here we prove the identity (3.21). Denote

$$
f(x, \theta):=\frac{1}{\cosh x+\cos \theta}, \quad \mathscr{I}(\lambda, \theta):=\frac{1}{2 \pi} \int_{-\infty}^{\infty} e^{-i \lambda x} f(x, \theta) d x, \quad 0<\theta<\pi .
$$

Since $f(x, \theta)=f(x+2 \pi i, \theta)$, we have

$$
\mathscr{I}_{s}(\lambda, \theta):=\frac{1}{2 \pi} \int_{-\infty+2 \pi i}^{\infty+2 \pi i} e^{-i \lambda x} f(x, \theta) d x=e^{2 \pi \lambda} \mathscr{I}(\lambda, \theta) .
$$


Further, the function $f(x, \theta)$ has two poles in the strip $\operatorname{Im} x \in(0,2 \pi)$, namely $x_{1}=i(\pi-\theta)$ and $x_{2}=i(\pi+\theta)$. Thus,

$$
\mathscr{I}(\lambda, \theta)=\mathscr{I}_{s}(\lambda, \theta)+\frac{1}{2 \pi} \sum_{k=1,2} \int_{l_{k}} e^{-i \lambda x} f(x, \theta) d x,
$$

where $l_{k}, k=1,2$, means a circle of a small radius centered at the point $x_{k}$, the integral over $l_{k}$ being taken in the counter-clockwise direction. Combining (A1) and (A2), we see that

$$
\mathscr{I}(\lambda, \theta)=\frac{1}{1-e^{2 \pi \lambda}} \sum_{k=1,2} \frac{1}{2 \pi} \int_{l_{k}} e^{-i \lambda x} f(x, \theta) d x .
$$

Taking into account the following expansion at the point $x_{k}$,

$$
\cosh x=-\cos \theta+\left(x-x_{k}\right) \sinh x_{k}+\cdots, \quad k=1,2,
$$

we obtain by the Cauchy theorem:

$$
\frac{1}{2 \pi} \int_{l_{k}} e^{-i \lambda x} f(x, \theta) d x=\frac{e^{-i \lambda x_{k}}}{-i \sinh x_{k}} .
$$

Thus, using the equalities $-i \sinh i z=\sin z$ and $\sin (\pi \pm \theta)=\mp \sin \theta$, we derive from (A3) that

$$
\mathscr{I}(\lambda, \theta)=\left(1-e^{2 \pi \lambda}\right)^{-1} \frac{e^{\lambda(\pi-\theta)}-e^{\lambda(\pi+\theta)}}{\sin \theta} .
$$

Now it is easy to show that the r.h.s. of this equality equals the 1.h.s. of (3.21).

II. Let us prove now the bound (3.22). To that end we use the following representation (see [5]) of the function $Q_{l}$ :

$$
Q_{l}(\cosh y)=2^{-\frac{1}{2}} \int_{y}^{\infty}(\cosh t-\cosh y)^{-\frac{1}{2}} e^{-\left(l+\frac{1}{2}\right) t} d t, \quad y \geqq 0 .
$$

Since

$$
\cosh t-\cosh y \geqq \int_{y}^{t} \sinh t^{\prime} d t^{\prime} \geqq(t-y) \sinh y,
$$

it follows from here that

$$
\begin{aligned}
Q_{l}(\cosh y) & \leqq(2 \sinh y)^{-\frac{1}{2}} \int_{y}^{\infty} \frac{e^{-\left(l+\frac{1}{2}\right) t}}{(t-y)^{\frac{1}{2}}} d t \\
& =(\sinh y)^{-\frac{1}{2}}(2 l+1)^{-\frac{1}{2}} e^{-\left(l+\frac{1}{2}\right) y} \int_{0}^{\infty} \frac{e^{-t}}{t^{\frac{1}{2}}} d t .
\end{aligned}
$$

Using the identity

$$
\int_{0}^{\infty} \frac{e^{-t}}{t^{\frac{1}{2}}} d t=2 \int_{0}^{\infty} e^{-t^{2}} d t=\pi^{\frac{1}{2}}
$$

we obtain the inequality

$$
Q_{l}(\cosh y) \leqq \pi^{\frac{1}{2}}(\sinh y)^{-\frac{1}{2}}(2 l+1)^{-\frac{1}{2}} e^{-\left(l+\frac{1}{2}\right) y} .
$$

To get (3.22) it remains to substitute $z=\cosh y$ and take into account that $\operatorname{arccosh} z=\log \left[z+\left(z^{2}-1\right)^{1 / 2}\right]$. 
Acknowledgements. This paper was written during my stay at the University Paris-Nord. It is a pleasure for me to thank Professor A. Martinez and the staff of Département de Mathématiques et Informatique for their hospitality. I am indebted to C.I.E.S. for financial support due to which my stay in France became possible.

I am grateful to Prof. M.Sh. Birman who has drawn my attention to the book [8]. I would also like to thank Prof. B. Simon for pointing out to me reference [1], and the referee for his useful critical remarks.

\section{References}

1. Albeverio, S., Høegh-Krohn, R., Wu, T.T.: A class of exactly solvable three-body quantum mechanical problems and the universal low energy behaviour. Phys. Lett. A 83, 3, 105-109 (1981)

2. Amado, R.D., Noble, J.V.: On Efimov's Effect: A new pathology of three-particle systems. Phys. Lett. B 35, 25-27 (1971); II, Phy. Lett D (3) 5, 1992-2002 (1972)

3. Birman, M.Sh., Solomyak, M.Z.: Spectral theory of selfadjoint operators in Hilbert space. Dordrecht: D. Reidel P.C., 1987

4. Efimov, V.: Energy levels arising from resonant two-body forces in a three-body system. Phys. Lett. B 33, 563-564 (1970)

5. Erdélyi, A. (Ed.): Higher transcendental functions. Vol. 2, New York, Toronto, London: McGraw-Hill 1953

6. Faddeev, L.D.: Mathematical aspects of the three-body problem in the quantum scattering theory. Trudy Mat. Inst. Steklov. 69 (1963) (Russian)

7. Faddeev, L.D., Merkuriev, S.P.: Scattering theory. Leningrad: Nauka, 1989 (Russian)

8. Grenander, U., Szegö, G.: Toeplitz forms and their applications. 2nd ed., New York: Chelsea P.C., 1984

9. Jensen, A., Kato, T.: Spectral properties of Schrödinger operators and time-decay of the wave functions. Duke. Math. J. 46, 5, 583-611 (1979)

10. Ovchinnikov, Yu.N., Sigal, I.M.: Number of bound states of three body systems and Efimov's effect. Ann. Phys. 123, 274-295 (1979)

11. Tamura, H.: The Efimov effect of three-body Schrödinger operators. J. Funct. Anal. 95, 433-459 (1991)

12. Yafaev, D.R.: On the theory of the discrete spectrum of the three-particle Schrödinger operator. Math. USSR-Sb. 23, 535-559 (1974)

13. Yafaev, D.R.: On the zero resonance for the Schrödinger equation. Notes of LOMI Seminars 51 (1975) (Russian)

Communicated by B. Simon 